\title{
Project Report \\ Pan-Resistome Characterization of Uropathogenic Escherichia coli and Klebsiella pneumoniae Strains Circulating in Uganda and Kenya, Isolated from 2017-2018
}

\author{
Arun Gonzales Decano ${ }^{1, *}$, Kerry Pettigrew ${ }^{1}$, Wilber Sabiiti ${ }^{1}$, Derek J. Sloan ${ }^{1}$, Stella Neema ${ }^{2}$, Joel Bazira ${ }^{3}$, \\ John Kiiru ${ }^{4}$, Hellen Onyango ${ }^{5}$, Benon Asiimwe ${ }^{6}$ and Matthew T. G. Holden ${ }^{1}$ \\ 1 School of Medicine, University of St Andrews, St Andrews KY16 8BQ, UK; \\ global.challenges@essb.eur.nl (K.P.); ws31@st-andrews.ac.uk (W.S.); djs26@st-andrews.ac.uk (D.J.S.); \\ mtgh@st-andrews.ac.uk (M.T.G.H.) \\ 2 Department of Sociology and Anthropology, Makerere University, Kampala P.O. Box 7062, Uganda; \\ sheisim@yahoo.com \\ 3 Faculty of Medicine, Mbarara University of Science and Technology, Mbarara P.O. Box 410, Uganda; \\ jbazira@gmail.com \\ 4 Centre of Microbiology Research, Kenya Medical Research Institute, Off Raila Odinga Way, \\ Nairobi P.O. Box 54840 00200, Kenya; kyirow@gmail.com \\ 5 Department of Medical Microbiology, Jomo Kenyatta University of Agriculture and Technology, \\ Nairobi P.O. Box 62 000, Kenya; hao1@st-andrews.ac.uk \\ check for \\ updates \\ Citation: Decano, A.G.; Pettigrew, K.; \\ 6 Department of Medical Microbiology, Makerere University College of Health Sciences, \\ Kampala P.O. Box 7062, Uganda; benon.asiimwe@gmail.com \\ * Correspondence: ad322@st-andrews.ac.uk
} Sabiiti, W.; Sloan, D.J.; Neema, S.; Bazira, J.; Kiiru, J.; Onyango, H.; Asiimwe, B.; Holden, M.T.G. Pan-Resistome Characterization of Uropathogenic Escherichia coli and Klebsiella pneumoniae Strains Circulating in Uganda and Kenya, Isolated from 2017-2018. Antibiotics 2021, 10, 1547. https://doi.org/ 10.3390/antibiotics10121547

Academic Editor: Seok Hoon Jeong

Received: 16 November 2021 Accepted: 7 December 2021 Published: 17 December 2021

Publisher's Note: MDPI stays neutral with regard to jurisdictional claims in published maps and institutional affiliations.

Copyright: (c) 2021 by the authors. Licensee MDPI, Basel, Switzerland. This article is an open access article distributed under the terms and conditions of the Creative Commons Attribution (CC BY) license (https:/ / creativecommons.org/licenses/by/ $4.0 /)$.

\begin{abstract}
Urinary tract infection (UTI) develops after a pathogen adheres to the inner lining of the urinary tract. Cases of UTIs are predominantly caused by several Gram-negative bacteria and account for high morbidity in the clinical and community settings. Of greater concern are the strains carrying antimicrobial resistance (AMR)-conferring genes. The gravity of a UTI is also determined by a spectrum of other virulence factors. This study represents a pilot project to investigate the burden of AMR among uropathogens in East Africa. We examined bacterial samples isolated in 2017-2018 from in- and out-patients in Kenya (KY) and Uganda (UG) that presented with clinical symptoms of UTI. We reconstructed the evolutionary history of the strains, investigated their population structure, and performed comparative analysis their pangenome contents. We found 55 Escherichia coli and 19 Klebsiella pneumoniae strains confirmed uropathogenic following screening for the prevalence of UTI virulence genes including fim $H, i u t A, f e o A / B / C, m r k D$, and foc. We identified 18 different sequence types in E. coli population while all K. pneumoniae strains belong to ST11. The most prevalent E. coli sequence types were ST131 (26\%), ST335/1193 (10\%), and ST10 (6\%). Diverse plasmid types were observed in both collections such as Incompatibility (IncF/IncH/IncQ1/IncX4) and Col groups. Pangenome analysis of each set revealed a total of 2862 and 3464 genes comprised the core genome of E. coli and K. pneumoniae population, respectively. Among these are acquired AMR determinants including fluoroquinolone resistance-conferring genes aac(3)- $\mathrm{Ib}-\mathrm{cr}$ and other significant genes: aad, tet, sul1, sul2, and cat, which are associated with aminoglycoside, tetracycline, sulfonamide, and chloramphenicol resistance, respectively. Accessory genomes of both species collections were detected several $\beta$-lactamase genes, $b l a_{\mathrm{CTX}-\mathrm{M}}, b l a_{\mathrm{TEM}}$ and $b l a_{\mathrm{OXA}}$, or $b l a_{\mathrm{NDM}}$. Overall, $93 \%$ are multi-drug resistant in the E. coli collection while $100 \%$ of the K. pneumoniae strains contained genes that are associated with resistance to three or more antibiotic classes. Our findings illustrate the abundant acquired resistome and virulome repertoire in uropathogenic E. coli and K. pneumoniae, which are mainly disseminated via clonal and horizontal transfer, circulating in the East African region. We further demonstrate here that routine genomic surveillance is necessary for high-resolution bacterial epidemiology of these important AMR pathogens.
\end{abstract}

Keywords: antimicrobial resistance; pangenome; lmic; public health 


\section{Introduction}

Antimicrobial resistance (AMR) has raised alarms as a global health threat. AMR is often fueled by misuse and abuse of antibiotics including self-medication [1,2] and unrestricted access to antimicrobial drugs [3-5], and is further accelerated by industrialization, poor waste disposal, and poor hygiene levels. AMR pathogens are frequently detected in food, clinical, and environmental settings in East Africa. Despite facing broad challenges, significant efforts have recently been put in place to curb AMR in East African countries. For instance, Kenya (KY) has adapted the National Action Plan that incorporates One Health measures to prevent AMR and is highly supported by multiple governmental policies (NAPCAR 2017) [6]. Similarly, an extensive evaluation of the AMR situation in Uganda (UG) was assessed by the Uganda National Academy of Sciences (UNAS) supported by the Global Antibiotic Resistance Partnership (GARP)-Uganda (UNAS 2015) [7]. High prevalence of multi-drug resistant bacteria particularly extended-spectrum beta-lactamase (ESBL)-producing strains is significantly recorded in both countries.

Urinary tract infection (UTI) develops after a pathogen's adherence to the inner lining of the urinary tract. UTIs occur among patients of all age groups and account for high morbidity in the clinical and community settings [8]. Following binding within the urinary tract, uropathogens either cause asymptomatic or commensal connection or severe disease. About $1 \%$ of the population have asymptomatic bacteriuria (ABU), wherein a pathogen $\left(\geq 10^{5} \mathrm{cfu} \mathrm{mL}{ }^{-1}\right)$ inhabits the tract without eliciting mucosal host response $[9,10]$. Infections in the lower urinary tract region (e.g., cystitis) are recognized by symptoms such as dysuria. Successful virulent strains can induce pyelonephritis where rapid immune response is mobilized via cytokine secretion and influx of immune cells. UTIs are either uncomplicated or complicated. Uncomplicated UTI cases are usually observed in patients who are otherwise healthy, while complicated UTIs are diagnosed in compromised patients (e.g., if they have anatomical or functional anomalies in their urinary tract or are under longterm catheterization) [11] Treatment of these complicated UTI cases is often confounded by AMR uropathogens usually caused by Gram-negative bacteria [12]. Uncomplicated UTIs are frequently caused by uropathogenic Escherichia coli (E. coli (UPEC)) while complicated cases might be caused by several pathogens such as Proteus mirabilis, Providencia stuartii, Morganella morganii, Klebsiella pneumoniae (K. pneumoniae), and Pseudomonas aeruginosa [8]. Recurrent UTI cases are also common, particularly when urinary tract anomalies linger, or treatment failed to kill resistant bacteria [13], leading to more severe type of infections. Due to the lack of active investigation of UTI cases in East Africa, particularly in the community, access to accurate data can be challenging.

An increasing number of studies have employed whole genome sequencing (WGS) and analyses for disease surveillance in both hospital and community settings [14-16]. The high-resolution genotyping that WGS provides allows one to investigate and describe the population structure and evolutionary history of the isolates, as well as tracing their spread. Outbreaks have been robustly detected and described using high-throughput methodologies designed for bacterial pathogens [17-20]. Comprehensive AMR gene databases and prediction tools are also available that help assess AMR gene content in whole genomes with high accuracy [21].

Here, we used WGS to investigate the prevalence of acquired AMR-conferring genes in E. coli and K. pneumoniae isolated from urine samples taken from patients in rural areas of KY and UG that presented UTI-like symptoms. Our analysis of AMR determinants was limited to those associated with the pan genome and mutation in core genes responsible for antibiotic resistance were not investigated. We further explored their phylogenetic relationships of the isolates collected with other currently circulating African and global strains. This study represents a pilot project of the HATUA consortium. HATUA stands for Holistic Approach to Unravel Antibiotic Resistance in East Africa and the team is comprised of researchers from different disciplines that aim to tackle the main drivers of AMR among uropathogens in East Africa. 


\section{Methods}

\subsection{Study Design and Patient Recruitment}

A total of $\mathrm{N}=150$ bacterial isolates were obtained from patients in $\mathrm{KY}(n=91)$ and UG $(n=59)$ presenting UTI-like symptoms, as part of a larger study. Ethical Review Board of University of St Andrews ethical approval, Approval code MD14548 and KY (KEMRI/SERU/P00112/3865) approved verbal consent taken from all the patients. Important patient data such as name, age, gender, location was recorded, and unique identification number were assigned to each patient.

\subsection{Library Preparation and Whole Genome Sequencing}

Bacterial genomic DNA for the isolates were extracted using the QIAxtractor (Qiagen, Valencia, CA, USA) according to the manufacturer's instructions. Library preparation was conducted according to the Illumina protocol and sequenced (96-plex) on an Illumina MiSeq platform (Illumina, San Diego, CA, USA) using 250 bp paired-end reads.

\subsection{Read Library Quality Control, Mapping and De Novo Genome Assembly}

Illumina MiSeq read libraries were rid of sequencing adapters and ambiguous bases using Fastp [22]. Sets that passed the quality filtering were de novo assembled using Unicycler v4.6 [23] pipeline in normal mode to merge contigs.

The read libraries were mapped to reference sequences using SMALT v7.6 (http: / / www.sanger.ac.uk/resources/software/SMALT / (accessed on 18 December 2019) [24] and the resulting SAM files were converted to BAM format, sorted and PCR duplicates removed using SAMtools v1.19 [25]. Strain TOP52_1721_U1 [26] was used the reference genome for the K. pneumoniae samples while the strain EC958 [27] was employed as the reference sequence for the $E$. coli population.

\subsection{Species Identification, Sero- and Sequence Typing, Genome Annotation and Screening for UTI Virulence/AMR Genes}

Prediction of bacterial species was carried out by uploading the assemblies on PathogenWatch website (https: / / pathogen.watch (accessed on 31 October 2019)) [28], which runs Speciator (https: / / gitlab.com/cgps/mash-speciator (accessed on 31 October 2019)) for its species assignment. Speciator employs Mash [29] to identify the most identical strain ( $\geq 90 \%$ identity) in a reference collection of complete genomes found in the NCBI RefSeq database (https:/ / www.ncbi.nlm.nih.gov/refseq/ (accessed on 31 October 2019)) [30]. The strains are then grouped according to their species designation and were screened for UTI pathogen determinants. Multi-locus sequence typing was performed by running SRST2 v.0.2.0 [31] based on the Achtman scheme [32] for E. coli and Pasteur [33] for K. pneumoniae isolates. Antigenic (O polysaccharide and $\mathrm{H}$ flagellin) profiles of $E$. coli samples were identified by employing Serotypefinder v.2.0 (https:/ / cge.cbs.dtu.dk/services/SerotypeFinder/ (accessed on 13 December 2019) [34] at 85\% ID threshold and 60\% minimum length.

Genome composition of the draft assemblies was assessed using Prokka v.1.10 [35] Acquired AMR genes were identified by aligning the genome sequences to the 2158 gene homolog subset of the Comprehensive Antibiotic Resistance Database (CARD) v. 3.0.8 (https: / / card.mcmaster.ca / (accessed on 08 November 2019)) [36] and BacWGSTdb 2.0 [37] Clustering based on the distribution of AMR genes among isolates was drawn using Phandango v.1.3.0 [38]. Plasmid and replicon typing was carried out by comparing the genomes against the PlasmidFinder database v. 2.1.1 [39] at 99\% identity threshold.

\subsection{Bacterial Sample Collection and Antimicrobial Susceptibility Testing}

To determine concordance between the AMR gene content and sample phenotype, antibiotic susceptibility testing and phenotypic detections of ESBL were performed by disc diffusion methods on a subset of $n=16$ isolates from KY. The tests were carried out according to CLSI (2016) guidelines [40]. Isolates were examined for the insusceptibility to 9 different classes of antibiotics including Penicillin (ampicillin (AMP)), Penicillin $+\beta$-lactamase 
inhibitors (ampicillin-clavulanic acid (AMC)), Chloramphenicol (Chloramphenicol (CHL)), Sulfonamide (Trimethoprim-sulfamethoxazole (SXT)) and Quinolones (nalidixic acid (NA)), and Fluoroquinolone (Ciprofloxacin (CIP)). Resistance to ESBL Cephalosphorins was also assessed by testing the strains with Ceftriaxone (CRO), Ceftazidime (CAZ), cefotaxime (CTX), and Cefepime (FEP) (Supplementary Table S1).

\subsection{Phylogenetic Reconstruction}

Phylogenetic relationships and sequence variations between the samples were determined by constructing phylogenetic trees based on their chromosomal single-nucleotide polymorphism (SNP)s. Mobile genetic elements (MGEs) were further excluded using an internal script. Non-recombinant SNPs were determined using ClonalframeML v. 1.12 [41] and were used to create a maximum-likelihood midpoint-rooted phylogeny using RAxML v8.0.19 [42] using a General Time Reversible + gamma (GTR + G) substitution model with 100 bootstraps. Phylogenies were visualized using iToL (https:/ /itol.embl.de/ (accessed on 20 January 2020)) [43] and FigTree v1.4.3 (http:/ / tree.bio.ed.ac.uk/software/figtree/ (accessed on 20 January 2020)) [44].

\subsection{Pangenome Analyses}

The resulting annotation files from Prokka v.1.10 [35] were used as the basis for generating a pangenome for each species set. This step was completed by running Roary v3.11.2 [45] with a 100\% BLAST v2.6.0 identity threshold using the MAFFT v7.3 setting [46]. Pangenome outputs were also used to assess the accessory genome composition of each bacterial population and as basis for reconstructing core genome phylogenies.

\section{Results}

\subsection{Patient and Bacterial Strain Profiles}

From the total of $\mathrm{N}=150$ strains, we collected from urine samples of patients, $n=81$ were identified as E. coli and $n=19$ were K. pneumoniae. The respondents were either to be admitted or visiting rural hospitals in KY and from clinics in the countryside of UG.

\subsection{Genomic and Pangenomic Characterization Confirmed the Virulence Factors Present in Uropathogenic E. coli and K. pneumoniae}

A subset $(n=55)$ from the total $n=81$ E. coli and all $n=19$ K. pneumoniae were confirmed uropathogenic following a thorough characterization of their pangenome contents. One thousand one hundred forty-four (1144) and 3464 core genes were found across the strains in E. coli and K. pneumoniae populations, respectively. These include known UTI virulence markers that are responsible for urinary tract (mucosal) surface binding (type 1 fimbrial adhesin-coding fimH) and colonization ( $m r k D ; K$. pneumoniae only), iron ( $\mathrm{Fe}(2+))$ transport $(f e o A / B / C)$, enterobactin synthase production (ent $B)$, formate transport $(f o c A)$, cell division $(z a p A)$, succinate-acetate/proton symport (satP), anaerobic sulfatase-maturation (chuR; found in 100\% and 95\% of E. coli and K. pneumoniae, respectively). Other important virulence genes were also found, albeit not conserved among all the isolates: iut $A$ (ferric aerobactin receptor: $44.6 \%$ in E. coli, $10 \%$ in K. pneumoniae), papA (fimbrial major pilin protein: E. coli only (41\%)), papD (import of $\mathrm{P}$ pilus subunits into the periplasm: $44.6 \%$ in E. coli, 10\% in K. pneumoniae), hlyE (hemolysin E: $80.4 \%$ in E. coli, 10\% in K. pneumoniae), fyuA (pesticin receptor: $73.2 \%$ in E. coli, 15\% in K. pneumoniae), kpsT (polysialic acid transport ATP-binding protein: E. coli only $(26.8 \%)$ ) and pic (serine protease pic autotransporter: E. coli only (5.4\%); Table 1$)$. 
Table 1. UTI virulence marker genes present in the pangenome of $\mathrm{N}=55$ E. coli and $\mathrm{N}=19 \mathrm{~K}$. pneumoniae isolates (including the reference genomes for each species collection). Proportion of the samples containing the gene are shown in count of strains with gene over the total strains and \% values.

\begin{tabular}{|c|c|c|c|}
\hline Gene & Protein Product & $\begin{array}{l}\text { Strain Count and \% in } \\
\text { E. coli Collection }\end{array}$ & $\begin{array}{l}\text { Strain Count and \% in } \\
\text { K. pneumoniae Collection }\end{array}$ \\
\hline fimH & Type 1 fimbrin D-mannose specific adhesin & $56 / 56(100)$ & $20 / 20(100)$ \\
\hline$f e o A / B / C$ & $\mathrm{Fe}(2+)$ transport protein $\mathrm{A} / \mathrm{B} / \mathrm{C}$ & $56 / 56(100)$ & $20 / 20(100)$ \\
\hline entB & Enterobactin synthase component B & $56 / 56(100)$ & $20 / 20(100)$ \\
\hline foc $A$ & Formate transporter & $56 / 56(100)$ & $20 / 20(100)$ \\
\hline$z a p A$ & Cell division protein & $56 / 56(100)$ & $20 / 20(100)$ \\
\hline satP & Succinate-acetate/proton symporter & $56 / 56(100)$ & $20 / 20(100)$ \\
\hline chuR & Anaerobic sulfatase-maturating enzyme & $56 / 56(100)$ & $19 / 20(95)$ \\
\hline$m r k D$ & Type 3 fimbrial adhesin & 0 & $20 / 20(100)$ \\
\hline$h l y E$ & Hemolysin E & $45 / 56(80.4)$ & $2 / 20(10)$ \\
\hline fyuA & Pesticin receptor & $41 / 56(73.2)$ & $3 / 20(15)$ \\
\hline iutA & Ferric aerobactin receptor & $25 / 56(44.6)$ & $2 / 20(10)$ \\
\hline papD & Import of $\mathrm{P}$ pilus subunits into the periplasm & $26 / 56(46.4)$ & $2 / 20(10)$ \\
\hline papA & Fimbrial major pilin protein & $23 / 56(41.1)$ & 0 \\
\hline kpsT & Polysialic acid transport ATP-binding protein & $15 / 56(26.8)$ & 0 \\
\hline pic & Serine protease pic autotransporter & $3 / 56(5.4)$ & 0 \\
\hline
\end{tabular}

\subsection{Prevalence of AMR Genes in E. coli and K. pneumoniae Uropathogens from $K Y$ and $U G$}

All $n=55$ E. coli and $n=19$ K. pneumoniae isolates harbored type 1 fimbrin. Among the UPEC, fimH30 was the most common allele, followed by fimH41; $n=4 / 55$ samples had type fim $\mathrm{H} 22$ and $n=2 / 55$ singleton were found with fim $\mathrm{H} 22$.

We further detected multiple acquired AMR-conferring elements in the genomes of the two species collections. Alignment of the sequences against CARD v.3.0.7 with $98-100 \%$ identity revealed that the $n=55 \mathrm{E}$. coli $(n=31$ from KY, $n=24$ from UG) were detected with the ciprofloxacin-conferring gene, mar $A$. Majority $(n=47 / 55)$ were also aminoglycoside resistant and harbors either $a a d A$ or $a a c\left(6^{\prime}\right)-I b /\left(3^{\prime}\right)-I b$ alleles or both. Only $n=11 / 55$ were not detected with resistant genes for ESBL cephalosphorins. Of the $n=44 / 55$ that produce ESBLs, $n=10 / 44$ had bla $a_{\text {CTX-M }}$ (allele type 15 or 88 ), $n=24 / 44$ had bla $a_{\text {TEM }}$ (type 30/2/220) and $n=9 / 44$ had $b l a_{\mathrm{OXA}-1 / 140}$ and $n=2 / 44$ (both from UG) had all 3 ESBL genes. Sulfonamide resistance genes were widely observed, $n=39$ had either sul1 only, sul2 only or sul 3 only, or both sul1 and sul2. Tetracycline resistance gene, tet $(A)$ was present in $n=34$ of 55 . Twenty-four $(\mathrm{N}=24 / 55)$ contained macrolide resistance-conferring $m p h A, n=9 / 55(n=6$ from $K Y, n=3$ from UG) harbored catB3 and were chloramphenicol resistant. Ninety-five percent $(95 \%, n=52 / 55)$ had at least one gene that codes for efflux pump proteins with $n=4 / 55$ having yojI-pmrF-emrR-bacA-acrS/B/E-msbA-evgA-kdpE-mdtP-ept $A$ cassette and $n=1 / 55$ containing a mixture of yojI, $p m r F, e m r R, b a c A, a c r S / B / E, m s b A, \operatorname{evg} A, k d p E, m d t P$, eptA, emtK, cpxA (Tables 2 and 3). Two KY isolates (71 and 72) were found to have the fluoroquinolone resistance-conferring gene $a a c\left(3^{\prime}\right)-\mathrm{Ib}-\mathrm{cr}$ while its variant $a a c\left(6^{\prime}\right)-\mathrm{Ib}$-cr was present in UG isolates BN19, BN38 and BN44 (Supplementary Figure S1a). 


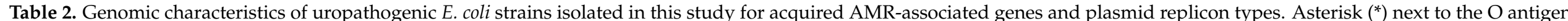
type means undefined; NF means not found; 'Yes' means the strain is either ESBL-producing or MDR; and 'No' means the sample is either non-ESBL or non-MDR.

\begin{tabular}{|c|c|c|c|c|c|c|c|c|c|c|c|c|c|c|c|c|c|c|}
\hline $\begin{array}{l}\text { Sample } \\
\text { Name }\end{array}$ & Serotype & $\begin{array}{c}\text { Sequenc } \\
\text { Type } \\
\text { Pasteur }\end{array}$ & $\begin{array}{c}\text { AMR } \\
\text { Phenotype }\end{array}$ & $\mathrm{fimH}$ & Aminoglycosides & Macrolide & Ciplrofloxacin & $\begin{array}{l}\beta \text {-Lactamase } \\
\text { Inhibitors/ESBL } \\
\text { Cephalosporins }\end{array}$ & Phenicols & Fluoroquinolones & Quinolone & Sulfonamide & Tetracycline & $\begin{array}{c}\text { Folate } \\
\text { Pathway } \\
\text { Inhibitors } \\
\end{array}$ & $\begin{array}{l}\text { Antibiotic Ef- } \\
\text { fulx/Regulation }\end{array}$ & ESBL & MDR & $\begin{array}{l}\text { Plasmid } \\
\text { Replicon }\end{array}$ \\
\hline 5 & $\mathrm{O} 25: \mathrm{H} 4$ & 131 & $\begin{array}{l}\text { AMP, CTX, } \\
\text { CHL, SXT, } \\
\text { CIP, NA } \\
\text { AMP, CTX, }\end{array}$ & $\mathrm{H} 30$ & $\begin{array}{l}\operatorname{aac}(3)-\mathrm{Ib}, \\
\operatorname{aad} A 5\end{array}$ & $m p h A$ & $\operatorname{mar} A$ & $b l a_{\mathrm{CTX}}-\mathrm{M}-27 / 99$ & $\mathrm{NF}$ & $\mathrm{NF}$ & $\mathrm{NF}$ & sul1, sul2 & $\operatorname{tet}(A)$ & dfrA17 & $\operatorname{gadW}$ & Yes & Yes & $\begin{array}{l}\text { IncFIA, } \\
\text { IncFII }\end{array}$ \\
\hline 6 & $\mathrm{O} 25: \mathrm{H} 4$ & 131 & $\begin{array}{l}\text { CRO, CHL, } \\
\text { SXT, CIP, } \\
\text { NA }\end{array}$ & $\mathrm{H} 30$ & $\begin{array}{l}\operatorname{aac}(3)-\mathrm{Ib}, \\
\operatorname{aad} A 5\end{array}$ & $m p h A$ & $\operatorname{mar} A$ & $b l a_{\mathrm{CTX}}-\mathrm{M}-27 / 99$ & $\mathrm{NF}$ & $\mathrm{NF}$ & $\mathrm{NF}$ & sul1, sul2 & $\operatorname{tet}(A)$ & dfrA17 & $\operatorname{gadW}$ & Yes & Yes & $\begin{array}{l}\text { IncFIA, } \\
\text { IncFII }\end{array}$ \\
\hline 7 & $\mathrm{O}: \mathrm{H} 5$ & 1193 & $\begin{array}{l}\text { CRO, CHL, } \\
\text { SXT, CIP, } \\
\text { NA }\end{array}$ & $\mathrm{H} 41$ & $\begin{array}{l}\text { aac(3)-Ib, } \\
\text { aadA17 }\end{array}$ & $m p h A$ & $\operatorname{mar} A$ & $b l a_{\text {TEM-220 }}$ & $\mathrm{NF}$ & $\mathrm{NF}$ & $\mathrm{NF}$ & $\mathrm{NF}$ & $\mathrm{NF}$ & $\mathrm{NF}$ & bacA, tolC, evgA $A$ & Yes & Yes & $\begin{array}{l}\text { IncQ1, } \\
\text { IncFIA, } \\
\text { Col1156, } \\
\text { Col(BS512) }\end{array}$ \\
\hline 8 & O75:H5 & 1193 & $\begin{array}{l}\text { AMP, FOX, } \\
\text { SXT, CIP, } \\
\text { NA }\end{array}$ & $\mathrm{H} 41$ & $\begin{array}{l}\text { aac(3)--Ib, } \\
\text { aadA17 }\end{array}$ & $m p h A$ & $\operatorname{mar} A$ & $b l a_{\mathrm{TEM}-220}$ & $\mathrm{NF}$ & NF & $\mathrm{NF}$ & $\mathrm{NF}$ & $\mathrm{NF}$ & $\mathrm{NF}$ & bac $A$, tolC, evg $A$ & Yes & Yes & $\begin{array}{c}\text { IncFIA, } \\
\text { IncQ1, } \\
\text { Col(BS5512), } \\
\text { Col156 }\end{array}$ \\
\hline 9 & O6:H1 & 73 & $\begin{array}{l}\text { AMP, FOX, } \\
\text { SXX, CII,, }\end{array}$ & H30 & $\mathrm{NF}$ & $\mathrm{NF}$ & $\operatorname{mar} A$ & $\mathrm{NF}$ & $\mathrm{NF}$ & NF & $\mathrm{NF}$ & $\mathrm{NF}$ & $\mathrm{NF}$ & $\mathrm{NF}$ & $\operatorname{mex} B$ & No & No & IncX1 \\
\hline 10 & O6:H1 & 73 & $\begin{array}{l}\text { NA } \\
\text { AMP, CTX }\end{array}$ & $\mathrm{H} 30$ & $\mathrm{NF}$ & $\mathrm{NF}$ & $\operatorname{mar} A$ & NF & NF & $\mathrm{NF}$ & NF & $\mathrm{NF}$ & NF & $\mathrm{NF}$ & $a c r B$ & No & No & $\mathrm{NF}$ \\
\hline 11 & $\mathrm{O} 25: \mathrm{H} 4$ & 131 & $\begin{array}{l}\text { AMP, CTX, } \\
\text { CRO, CHL, } \\
\text { SXT, CIP, } \\
\text { NA }\end{array}$ & $\mathrm{H} 30$ & $\begin{array}{l}\operatorname{aac}(3)-\mathrm{Ib}, \\
\text { aad } A 5\end{array}$ & $m p h A$ & $\operatorname{mar} A$ & $b^{b l a}{ }_{\mathrm{CTX}}-\mathrm{M}-27 / 99$ & $\mathrm{NF}$ & NF & $\mathrm{NF}$ & sul1, sul2 & $\operatorname{tet}(A)$ & $\mathrm{NF}$ & $\begin{array}{l}\underset{\operatorname{acrB}, \operatorname{gad} W,}{ }, \mathrm{pmrF}\end{array}$ & Yes & Yes & $\begin{array}{l}\text { IncFIA, } \\
\text { IncFII, } \\
\text { Col156 }\end{array}$ \\
\hline 12 & $\mathrm{O} 25: \mathrm{H} 4$ & 131 & $\begin{array}{l}\text { AMP, CTX, } \\
\text { CRO, SXT, } \\
\text { CII, NA }\end{array}$ & $\mathrm{H} 30$ & $\begin{array}{l}\operatorname{aac}(3)-\mathrm{Ib}, \\
\operatorname{aad} A 5\end{array}$ & $m p h A$ & $\operatorname{mar} A$ & ${ }^{b l a} a_{\text {CTX-M-27/99 }}$ & $\mathrm{NF}$ & $\mathrm{NF}$ & $\mathrm{NF}$ & sul1, sul2 & $\operatorname{tet}(A)$ & $\mathrm{NF}$ & $\begin{array}{l}\operatorname{acrB,} \text {, gad W, } \\
\quad \text { pmrF }\end{array}$ & Yes & Yes & $\begin{array}{l}\text { IncFIA, } \\
\text { IncFII, } \\
\text { Col156 }\end{array}$ \\
\hline 13 & $\mathrm{O} 55: \mathrm{H} 7$ & 335 & $\begin{array}{l}\text { AMP, SXT, } \\
\text { NA }\end{array}$ & $\mathrm{H} 30$ & $\operatorname{aac}(3)-\mathrm{Ib}$ & $\mathrm{NF}$ & $\operatorname{mar} A$ & $b l a_{\mathrm{TEM}-220}$ & $\mathrm{NF}$ & $\mathrm{NF}$ & $\mathrm{NF}$ & sul1 & $\operatorname{tet}(A)$ & $d f r A 7$ & $\underset{\operatorname{gevg} A, c p x A}{\operatorname{egd} W}$ & Yes & Yes & IncQ1 \\
\hline 14 & $\mathrm{O} 55: \mathrm{H7}$ & 335 & $\begin{array}{l}\text { AMP, SXT, } \\
\text { NA }\end{array}$ & $\mathrm{H} 30$ & $a a c(3)-\mathrm{Ib}$ & $\mathrm{NF}$ & marA & $b l a_{\text {TEM-220 }}$ & $\mathrm{NF}$ & $\mathrm{NF}$ & $\mathrm{NF}$ & sul1 & $\operatorname{tet}(A)$ & $d f r A 7$ & $\operatorname{evg} A, c p x A$ & Yes & Yes & IncQ1 \\
\hline 15 & $\mathrm{O} 55: \mathrm{H} 7$ & 335 & $\begin{array}{l}\text { AMP, CTX, } \\
\text { SXT, NA }\end{array}$ & $\mathrm{H} 30$ & $\operatorname{aac}(3)-\mathrm{Ib}$ & $\mathrm{NF}$ & $\operatorname{mar} A$ & $b a_{\mathrm{TEM}-220}$ & $\mathrm{NF}$ & $\mathrm{NF}$ & NF & sul1 & $\operatorname{tet}(A)$ & $d f r A 7$ & $\operatorname{evg} A, c p x A$ & Yes & Yes & IncQ1 \\
\hline 16 & $\mathrm{O} 55: \mathrm{H7}$ & 335 & $\begin{array}{l}\text { AMP, SXT, } \\
\text { NA }\end{array}$ & $\mathrm{H} 30$ & $\operatorname{aac}(3)-\mathrm{Ib}$ & $\mathrm{NF}$ & $\operatorname{mar} A$ & $b l a_{\mathrm{TEM}-220}$ & NF & $\mathrm{NF}$ & $\mathrm{NF}$ & sul1 & $\operatorname{tet}(A)$ & $d f r A 7$ & $\operatorname{evg} A, \operatorname{cpx} A$ & Yes & Yes & IncQ1 \\
\hline 20 & $\mathrm{O} 25: \mathrm{H} 4$ & 131 & $\begin{array}{l}\text { AMP, CTX, } \\
\text { FOX, SXI, } \\
\text { CIP, NA }\end{array}$ & $\mathrm{H} 30$ & $\begin{array}{l}\operatorname{aac}(3)-\mathrm{Ib}, \\
\operatorname{aad} A 5\end{array}$ & $m p h A$ & $\operatorname{mar} A$ & $b l a_{\mathrm{CTX}}-\mathrm{M}-27 / 99$ & $\mathrm{NF}$ & $\mathrm{NF}$ & $\mathrm{NF}$ & sul1, sul2 & $\operatorname{tet}(A)$ & dfrA17 & $\operatorname{acrB}, \operatorname{gad} W$ & Yes & Yes & $\begin{array}{l}\text { IncFIA, } \\
\text { IIcFII, } \\
\text { Col156 } \\
\text { IncO1 }\end{array}$ \\
\hline 21 & $\mathrm{O} 75: \mathrm{H} 5$ & 1193 & $\begin{array}{l}\text { AMP, FOX, } \\
\text { SXX, CIII, } \\
\text { NA }\end{array}$ & $\mathrm{H} 41$ & $a a c(3)-\mathrm{Ib}$ & $m p h A$ & $\operatorname{mar} A$ & $b l a_{\mathrm{TEM}-220}$ & $\mathrm{NF}$ & $\mathrm{NF}$ & $\mathrm{NF}$ & $\mathrm{NF}$ & NF & dfrA17 & $\operatorname{evg} A$, tolc, bac $A$ & Yes & Yes & $\begin{array}{l}\text { IncQ1, } \\
\text { IncIIA, } \\
\text { Coll156, } \\
\text { Col(BS512) }\end{array}$ \\
\hline 23 & $\mathrm{O} 25: \mathrm{H} 4$ & 131 & $\begin{array}{l}\text { AMP, CTX, } \\
\text { CRO, CAZ, } \\
\text { FEP, CHL, } \\
\text { SXT, CII, } \\
\text { NA }\end{array}$ & $\mathrm{H} 30$ & $\begin{array}{l}\operatorname{aac}(3)-\mathrm{Ib}, \\
\operatorname{aad} A 5\end{array}$ & $m p h A$ & $\operatorname{mar} A$ & $b l a_{\mathrm{CTX}}-\mathrm{M}-27 / 99$ & $\mathrm{NF}$ & $\mathrm{NF}$ & $\mathrm{NF}$ & sul1, sul2 & $\operatorname{tet}(A)$ & dfra17 & $a c r B, g a d W$ & Yes & Yes & $\begin{array}{l}\text { IncFIA, } \\
\text { IncFII, } \\
\text { Col156 }\end{array}$ \\
\hline 24 & $\mathrm{O} 25: \mathrm{H} 4$ & 131 & $\begin{array}{l}\text { AMP, CTX, } \\
\text { CRO, SXT, } \\
\text { CIP, NA }\end{array}$ & $\mathrm{H} 30$ & $\begin{array}{l}\operatorname{aac}(3)-\mathrm{Ib}, \\
\operatorname{aad} A 5\end{array}$ & $m p h A$ & $\operatorname{mar} A$ & $b l a_{\mathrm{CTX}}-\mathrm{M}-27 / 99$ & $\mathrm{NF}$ & $\mathrm{NF}$ & $\mathrm{NF}$ & sul1, sul2 & $\operatorname{tet}(A)$ & dfra17 & acrB, gadW & Yes & Yes & $\begin{array}{l}\text { IncFIA, } \\
\text { IncFII, } \\
\text { Col156 } \\
\text { Incol, }\end{array}$ \\
\hline 60 & $\mathrm{O}^{*}: \mathrm{H} 5$ & 1193 & ND & $\mathrm{H} 41$ & $\begin{array}{l}\operatorname{aac}(3)-\mathrm{Ib}, \\
\operatorname{aad} A 5\end{array}$ & $m p h A$ & $\operatorname{mar} A$ & $b a_{\mathrm{TEM}-220}$ & $\mathrm{NF}$ & $\mathrm{NF}$ & $\mathrm{NF}$ & $\mathrm{NF}$ & $\mathrm{NF}$ & dfrA17 & acrs, bacA, tolC & Yes & Yes & 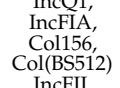 \\
\hline 63 & $\mathrm{O} 25: \mathrm{H} 4$ & 131 & ND & $\mathrm{H} 30$ & $\begin{array}{l}\operatorname{aac}(3)-\mathrm{Ib}, \\
\text { aadA5 }\end{array}$ & $m p h A$ & $\operatorname{mar} A$ & $b l a_{\mathrm{CTX}}-\mathrm{M}-27 / 99$ & $\mathrm{NF}$ & $\mathrm{NF}$ & $\mathrm{NF}$ & sul1, sul2 & $\operatorname{tet}(A)$ & dfra17 & $\operatorname{gad} W$ & Yes & Yes & $\begin{array}{l}\text { IncFII, } \\
\text { Col156, } \\
\text { IncFIA }\end{array}$ \\
\hline 64 & $\mathrm{O} 25: \mathrm{H} 4$ & 131 & ND & $\mathrm{H} 30$ & $\begin{array}{l}\operatorname{aac}(3)-\mathrm{Ib}, \\
\operatorname{aadA5}\end{array}$ & $m p h A$ & $\operatorname{mar} A$ & ${ }^{b l a} a_{\mathrm{CTX}-\mathrm{M}-27 / 99}$ & $\mathrm{NF}$ & $\mathrm{NF}$ & $\mathrm{NF}$ & sul1, sul2 & $\operatorname{tet}(A)$ & dfra17 & $g a d W$ & Yes & Yes & $\begin{array}{l}\text { IncFIA, } \\
\text { IncFII, } \\
\text { Coll156 }\end{array}$ \\
\hline 67 & $\mathrm{O} 25: \mathrm{H} 4$ & 131 & $\mathrm{ND}$ & $\mathrm{H} 30$ & $\begin{array}{l}\operatorname{aac}(3)-\mathrm{Ib}, \\
\text { aadA5 }\end{array}$ & $m p h A$ & marA & $\begin{array}{l}b l a_{\mathrm{CTX}}-\mathrm{M}-15 / 88 \\
b l a_{\mathrm{OX}-1 / 140}\end{array}$ & catB3 & $\mathrm{NF}$ & $\mathrm{NF}$ & sul1 & $\operatorname{tet}(A)$ & dfrA17 & acrS, gadW & Yes & Yes & IncFIA \\
\hline 68 & $\mathrm{O} 89: \mathrm{H} 4$ & 44 & $\mathrm{ND}$ & $\mathrm{H} 54$ & $\begin{array}{l}\operatorname{aac}(3)-\mathrm{Ib}, \\
\operatorname{aad} A 5\end{array}$ & $m p h A$ & $\operatorname{mar} A$ & $\begin{array}{c}b l_{\text {CTX-M-15/88 }} \\
\text { bla }_{\text {OXA-1/140 }}\end{array}$ & catB3 & $\mathrm{NF}$ & $\mathrm{NF}$ & sul1 & $\operatorname{tet}(A)$ & dfrA17 & acrs, gadW & Yes & Yes & $\begin{array}{l}\text { IncFIA, } \\
\text { IncFII }\end{array}$ \\
\hline
\end{tabular}


Table 2. Cont.

\begin{tabular}{|c|c|c|c|c|c|c|c|c|c|c|c|c|c|c|c|c|c|c|}
\hline $\begin{array}{l}\text { Sample } \\
\text { Name }\end{array}$ & Serotype & $\begin{array}{c}\text { Sequenc } \\
\text { Type } \\
\text { Pasteur }\end{array}$ & $\begin{array}{c}\text { AMR } \\
\text { Phenotype }\end{array}$ & fimH & Aminoglycosides & Macrolide & Ciplrofloxacin & $\begin{array}{c}\beta \text {-Lactamase } \\
\text { IIhibitiors/ESBL } \\
\text { Cephalosporins } \\
\end{array}$ & Phenicols & Fluoroquinolones & Quinolone & Sulfonamide & Tetracycline & $\begin{array}{c}\text { Folate } \\
\text { Pathway } \\
\text { Inhibitors }\end{array}$ & $\begin{array}{l}\text { Antibiotic Ef- } \\
\text { fulx/Regulation }\end{array}$ & ESBL & MDR & $\begin{array}{l}\text { Plasmid } \\
\text { Replicon }\end{array}$ \\
\hline 71 & $\mathrm{O} 25: \mathrm{H} 4$ & 131 & ND & $\mathrm{H} 30$ & aad $A 5$ & $\mathrm{NF}$ & $\operatorname{mar} A$ & $\begin{array}{c}b l a_{\text {CTX-M-15/88, }} \\
b l a_{\text {OXA-1/140 }}\end{array}$ & catB3 & $\operatorname{aac}(3)-\mathrm{lb}-\mathrm{cr}$ & QnrB2 & sul1 & $\operatorname{tet}(A)$ & dfrA17 & $\operatorname{gadW}$ & Yes & Yes & $\begin{array}{l}\text { IncFIA, } \\
\text { IncFII, } \\
\text { IncY, }\end{array}$ \\
\hline 72 & $\mathrm{O} 25: \mathrm{H} 4$ & 131 & ND & $\mathrm{H} 30$ & aadA5 & $\mathrm{NF}$ & $\operatorname{mar} A$ & $\begin{array}{l}b l a_{\text {CTX-M-15/88, }} \\
b l a_{O} \text { OXA-15 }\end{array}$ & catB3 & $\operatorname{aac}(3)-\mathrm{Ib}-\mathrm{cr}$ & $\mathrm{NF}$ & sul2 & $\mathrm{NF}$ & dfrA14 & $\operatorname{gadW}$ & Yes & Yes & $\begin{array}{l}\text { IncFIA, } \\
\text { IncFII }\end{array}$ \\
\hline 73 & $\mathrm{O} 25: \mathrm{H} 4$ & 131 & ND & $\mathrm{H} 30$ & $\begin{array}{l}\text { aac(3)-Ib, } \\
\text { aadA5 }\end{array}$ & $m p h A$ & $\operatorname{mar} A$ & $\begin{array}{c}b_{\text {CTX-M-1 }} \text { CX/150 } \\
\text { bla }\end{array}$ & catB3 & $\mathrm{NF}$ & $\mathrm{NF}$ & sul1 & $\operatorname{tet}(A)$ & dfrA17 & acrS, gadW & Yes & Yes & $\begin{array}{l}\text { IncFIA, } \\
\text { IncFII }\end{array}$ \\
\hline 74 & $\mathrm{O} * \mathrm{H} 6$ & 648 & ND & $\mathrm{H} 30$ & $\begin{array}{l}\text { aac(3)-Ib, } \\
\text { aadA5 }\end{array}$ & $m p h A$ & $\operatorname{mar} A$ & $\begin{array}{l}b_{\text {CTX-M-15/8 }} \\
b^{\prime} a_{\text {OXA }-1 / 140}\end{array}$ & catB3 & $\mathrm{NF}$ & $\mathrm{NF}$ & sul1 & $\operatorname{tet}(A)$ & dfrA17 & $\operatorname{acr} S, \operatorname{gad} W$ & Yes & Yes & $\begin{array}{l}\text { IncFIA, } \\
\text { IncFII }\end{array}$ \\
\hline 87 & $\mathrm{O}: \mathrm{H} 4$ & 131 & ND & $\mathrm{H} 30$ & $\begin{array}{l}\text { aac(3)-Ib, } \\
\text { aadA5 }\end{array}$ & $m p h A$ & $\operatorname{mar} A$ & $b l a_{\mathrm{CTX}} \mathrm{M}-\mathrm{M}-27 / 99$ & $\mathrm{NF}$ & $\mathrm{NF}$ & $\mathrm{NF}$ & sul1 & $\operatorname{tet}(A)$ & dfrA17 & $\operatorname{gadW}$ & Yes & Yes & $\begin{array}{l}\text { IncFIA, } \\
\text { IncFII }\end{array}$ \\
\hline 97 & $\mathrm{O} 25: \mathrm{H} 4$ & 131 & ND & H30 & $\begin{array}{l}\operatorname{aac}(3)-\mathrm{Ib}, \\
\operatorname{aad} A 5\end{array}$ & $m p h A$ & $\operatorname{mar} A$ & $\mathrm{bla}_{\mathrm{CTX}-\mathrm{M}-27 / 99}$ & $\mathrm{NF}$ & $\mathrm{NF}$ & $\mathrm{NF}$ & sul1 & $\operatorname{tet}(A)$ & dfrA17 & $\operatorname{gadW}$ & Yes & Yes & $\begin{array}{l}\text { InchIA, } \\
\text { IncFIII, } \\
\text { Col156 }\end{array}$ \\
\hline 102 & $\mathrm{O} * \mathrm{H} 7$ & 335 & $\mathrm{ND}$ & $\mathrm{H} 30$ & $\operatorname{aac}(3)-\mathrm{Ib}$ & $\mathrm{NF}$ & marA & $b l a$ TEM-220 & NF & $\mathrm{NF}$ & $\mathrm{NF}$ & sul1 & $\operatorname{tet}(A)$ & $d f r A 7$ & $\operatorname{cpXxA}, \operatorname{evg} A$ & Yes & Yes & IncQ1 \\
\hline 103 & $\mathrm{O} 25: \mathrm{H} 4$ & 131 & $\mathrm{ND}$ & $\mathrm{H} 30$ & $\begin{array}{l}\operatorname{aac}(3)-\mathrm{Ib}, \\
\operatorname{aad} A 5\end{array}$ & $m p h A$ & $\operatorname{mar} A$ & $b l a_{\text {CTX-M-27/99 }}$ & $\mathrm{NF}$ & $\mathrm{NF}$ & $\mathrm{NF}$ & sul2 & $\operatorname{tet}(A)$ & dfrA17 & NF & Yes & Yes & $\begin{array}{l}\text { IncFIA, } \\
\text { IncFIII, } \\
\text { Col15 }\end{array}$ \\
\hline 105 & O55:H7 & 335 & ND & $\mathrm{H} 30$ & $\operatorname{aac}(3)-\mathrm{Ib}$ & $\mathrm{NF}$ & $\operatorname{mar} A$ & $b l a_{\mathrm{TEM}-220}$ & $\mathrm{NF}$ & $\mathrm{NF}$ & $\mathrm{NF}$ & sul1 & $\operatorname{tet}(A)$ & dfrA7 & cpxA, evg $A$, acr $B$ & Yes & Yes & IncQ1 \\
\hline 106 & $\mathrm{O} * \mathrm{H} 5$ & 1193 & ND & $\mathrm{H} 41$ & $\operatorname{aac}(3)-\mathrm{Ib}$ & $m p h A$ & $\operatorname{mar} A$ & $b l a_{\mathrm{TEM}-220}$ & $\mathrm{NF}$ & $\mathrm{NF}$ & $\mathrm{NF}$ & $\mathrm{NF}$ & $\mathrm{NF}$ & dfrA17 & $\begin{array}{c}\text { evg } A \text {, tolC, } \\
m p h A\end{array}$ & Yes & Yes & $\begin{array}{l}\text { IncQ1, } \\
\text { IncFIA, } \\
\text { Col156 }\end{array}$ \\
\hline BN1 & O75:H5 & 1193 & $\mathrm{ND}$ & $\mathrm{H} 41$ & $\operatorname{aac}(3)-\mathrm{Ib}$ & $m p h A$ & marA & $b a_{\mathrm{TEM}-220}$ & NF & $\mathrm{NF}$ & $\mathrm{NF}$ & NF & $\mathrm{NF}$ & dfrA17 & bacA, tolC, evg $A$ & Yes & Yes & $\begin{array}{l}\text { IncQ1, } \\
\text { IncFIA }\end{array}$ \\
\hline BN12 & $\mathrm{O} * \mathrm{H} 9$ & 410 & ND & $\mathrm{H} 41$ & $\begin{array}{c}\operatorname{aad} A 9, \\
\operatorname{aac}(3)-\mathrm{Ib}\end{array}$ & $m p h A$ & $\operatorname{mar} A$ & $b l a_{\text {TEM-220 }}$ & $\mathrm{NF}$ & $\mathrm{NF}$ & $\mathrm{NF}$ & $\mathrm{NF}$ & $\mathrm{NF}$ & dfrA17 & emrR & Yes & Yes & $\begin{array}{l}\text { IncQ1, } \\
\text { IncFIA }\end{array}$ \\
\hline BN19 & $\mathrm{O} 25: \mathrm{H} 4$ & 131 & ND & $\mathrm{H} 30$ & $\begin{array}{c}\operatorname{aad} A 5, \\
\operatorname{aac}(3)-\mathrm{Ib}, \\
\operatorname{aac}(3)-\mathrm{Iic} / \mathrm{d} / \mathrm{e}\end{array}$ & $\mathrm{NF}$ & $\operatorname{mar} A$ & 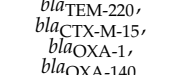 & catB3 & $a a c\left(6^{\prime}\right)-\mathrm{Ib}-\mathrm{cr}$ & $\mathrm{NF}$ & sul1, sul2 & $\mathrm{NF}$ & $\mathrm{NF}$ & $k d p E, g a d W$ & Yes & Yes & $\begin{array}{l}\text { IncFIA, } \\
\text { IncFII }\end{array}$ \\
\hline BN25 & O6:H11 & 48 & ND & H41 & $\begin{array}{l}\operatorname{aad} A 5,5,5 \\
\operatorname{aac}(3)-\mathrm{Ib}\end{array}$ & $\mathrm{NF}$ & $\operatorname{mar} A$ & $\mathrm{NF}$ & $\mathrm{NF}$ & $\mathrm{NF}$ & $\mathrm{NF}$ & sul2 & NF & dfrA17 & 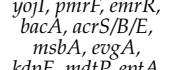 & No & Yes & IncHI2A \\
\hline BN26 & O9:H19 & $162^{*}$ & ND & $\mathrm{H} 30$ & $\mathrm{NF}$ & $\mathrm{NF}$ & $\operatorname{mar} A$ & $\mathrm{NF}$ & $\mathrm{NF}$ & $\mathrm{NF}$ & $\mathrm{NF}$ & $\mathrm{NF}$ & $\operatorname{tet}(A)$ & $\mathrm{NF}$ & 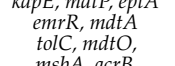 & No & Yes & $\mathrm{NF}$ \\
\hline BN27 & $\mathrm{O}: \mathrm{H} 2$ & 165 & ND & H54 & $\begin{array}{l}\operatorname{aac}\left(6^{\prime}\right)-\mathrm{Ib} 7, \\
\text { aac(3)-[-I, } \\
\text { aad } A 9\end{array}$ & $\mathrm{NF}$ & $\operatorname{mar} A$ & $b a_{\mathrm{TEM}-220}$ & $\mathrm{NF}$ & $\mathrm{NF}$ & qurS1 & sul3 & $\operatorname{tet}(A)$ & $\mathrm{NF}$ & $\begin{array}{c}m s b A, \text { acrB, } \\
\text { baes/R, acrD, } \\
\text { gad } \mathrm{d}, \text { ecogA } A \\
\text { pmrF }\end{array}$ & Yes & Yes & $\mathrm{NF}$ \\
\hline BN3 & O18:H49 & 212 & ND & $\mathrm{H} 30$ & 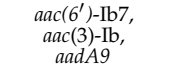 & $\mathrm{NF}$ & $\operatorname{mar} A$ & NF & $\mathrm{NF}$ & $\mathrm{NF}$ & $\mathrm{NF}$ & sul1 & $\operatorname{tet}(A)$ & dfrA15 & emrR & No & Yes & NF \\
\hline BN37 & O8:H17 & 10 & ND & H41 & $\begin{array}{l}\operatorname{aac}(3)-\mathrm{Ib}, \\
\operatorname{aad} A 5\end{array}$ & $\mathrm{NF}$ & $\operatorname{mar} A$ & $b l a_{\text {TEM-220 }}$ & $\mathrm{NF}$ & $\mathrm{NF}$ & $\mathrm{NF}$ & sul2 & $\operatorname{tet}(A)$ & dfrA15 & 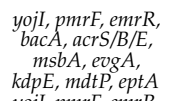 & Yes & Yes & $\begin{array}{l}\text { IncFIA, } \\
\text { IncFII }\end{array}$ \\
\hline BN38 & O89:H10 & 617 & ND & H41 & $\mathrm{NF}$ & $\mathrm{NF}$ & $\operatorname{mar} A$ & $\begin{array}{c}b l a_{\text {CTX-M-15/88, }} \\
b l a_{\text {OXA-1/ } 1 / 140}\end{array}$ & catB3 & $\operatorname{aac}\left(6^{\prime}\right)-\mathrm{Ib}-\mathrm{cr}$ & $\mathrm{NF}$ & sul1, sul2 & $\mathrm{NF}$ & $\mathrm{NF}$ & 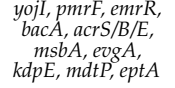 & Yes & Yes & $\begin{array}{l}\text { IncFIA, } \\
\text { IncFII }\end{array}$ \\
\hline BN41 & $\mathrm{O} 171: \mathrm{H} 21$ & 155 & ND & $\mathrm{H} 30$ & $\begin{array}{c}\operatorname{aac}\left(6^{\prime}\right)-\mathrm{Ib} 7, \\
\operatorname{aad} A 9\end{array}$ & $\mathrm{NF}$ & $\operatorname{mar} A$ & $b l a_{\mathrm{TEM}-220}$ & $\mathrm{NF}$ & $\mathrm{NF}$ & NF & sul2 & $\operatorname{tet}(A)$ & $\mathrm{NF}$ & emrR & Yes & Yes & $\begin{array}{l}\text { IncHI1A, } \\
\text { IncHIIB, } \\
\text { IncFIA, } \\
\text { Incl. }\end{array}$ \\
\hline BN42 & $\mathrm{O} 29: \mathrm{H} 8$ & 448 & ND & $\mathrm{H} 30$ & $\begin{array}{l}\operatorname{aad} A 13, \\
\operatorname{aac}(3)-\mathrm{Ib}\end{array}$ & $\mathrm{NF}$ & $\operatorname{mar} A$ & $b l a_{\text {CTX-M-5 }}$ & $\mathrm{NF}$ & $\mathrm{NF}$ & $\mathrm{NF}$ & sul2 & $\mathrm{NF}$ & dfrA14 & $e m r R, m p h A$ & Yes & Yes & $\begin{array}{l}\text { IncFII, } \\
\text { IncIIA } \\
\text { IncX4 }\end{array}$ \\
\hline $\mathrm{BN} 43$ & $\mathrm{O} * \mathrm{H} 4$ & 6161 & ND & $\mathrm{H} 30$ & $\mathrm{NF}$ & $\mathrm{NF}$ & $\operatorname{mar} A$ & $\mathrm{NF}$ & NF & NF & $\mathrm{NF}$ & sul2 & $\mathrm{NF}$ & dfrA14 & baes & No & Yes & $\mathrm{NF}$ \\
\hline
\end{tabular}


Table 2. Cont

\begin{tabular}{|c|c|c|c|c|c|c|c|c|c|c|c|c|c|c|c|c|c|c|}
\hline $\begin{array}{l}\text { Sample } \\
\text { Name }\end{array}$ & Serotype & $\begin{array}{c}\text { Sequenc } \\
\text { Type } \\
\text { Pasteur }\end{array}$ & $\begin{array}{l}\text { AMR } \\
\text { Phenotype }\end{array}$ & fimH & Aminoglycosides & Macrolide & Ciplrofloxacin & $\begin{array}{c}\text { B-Lactamase } \\
\text { Inhibitors/ESBL } \\
\text { Cephalosporins }\end{array}$ & Phenicols & Fluoroquinolones & Quinolone & Sulfonamide & Tetracycline & $\begin{array}{c}\text { Folate } \\
\text { Pathway } \\
\text { Inhibitors }\end{array}$ & $\begin{array}{l}\text { Antibiotic Ef- } \\
\text { fulx/Regulation }\end{array}$ & ESBL & MDR & $\begin{array}{l}\text { Plasmid } \\
\text { Replicon }\end{array}$ \\
\hline BN44 & $\mathrm{O}: \mathrm{H} 9$ & 410 & ND & $\mathrm{H} 41$ & $\begin{array}{l}\operatorname{aac}\left(6^{\prime}\right) \text {-Ib7, } \\
\text { aad } A 9\end{array}$ & $m p h A$ & $\operatorname{mar} A$ & $\begin{array}{c}b l a_{\text {CTX-M-5 }} \\
b a_{O X X A-1} \\
b a_{\text {TEM-30/220 }}\end{array}$ & catB3 & $\operatorname{aac}\left(6^{\prime}\right)$-Ib-cr & $\mathrm{NF}$ & $\mathrm{NF}$ & $\mathrm{NF}$ & $\mathrm{NF}$ & $e m r R$ & Yes & Yes & $\begin{array}{l}\text { IncFIA, } \\
\text { IncQ1 }\end{array}$ \\
\hline BN47 & $\mathrm{O} 89: \mathrm{H} 9$ & 10 & $\mathrm{ND}$ & H54 & $\begin{array}{l}\operatorname{acr} D / \mathrm{E} / \mathrm{F}, \\
\operatorname{aad} A 9, \\
\operatorname{aac}(3)-\mathrm{Ib}, \\
\operatorname{aac}\left(6^{\prime}\right)-\mathrm{Ib} 7\end{array}$ & $\mathrm{NF}$ & $\operatorname{mar} A$ & $b l a_{\text {TEM-220 }}$ & $\mathrm{NF}$ & $\mathrm{NF}$ & $\mathrm{NF}$ & sul1 & $\mathrm{NF}$ & dfrA1 & $\begin{array}{c}\operatorname{gadX}, t o l C, \\
m d t F / N / O, e m r K\end{array}$ & Yes & Yes & $\begin{array}{l}\text { Col440II, } \\
\text { IncQ1 }\end{array}$ \\
\hline $\mathrm{BN} 48$ & O17:H11 & $\mathrm{NF}$ & $\mathrm{ND}$ & $\mathrm{H} 41$ & $\operatorname{aac}(3)-\mathrm{Ib}$ & $\mathrm{NF}$ & $\operatorname{mar} A$ & $b l a_{\text {TEM- }-7 / 75 / 177}$ & $\mathrm{NF}$ & $\mathrm{NF}$ & $\mathrm{NF}$ & sul1 & $\mathrm{NF}$ & $d f r A 7$ & $\begin{array}{c}a x y Y \\
y o j I, \text { pmrF, emrR, }\end{array}$ & Yes & Yes & IncQ1 \\
\hline BN49 & O45:H11 & 10 & $\mathrm{ND}$ & $\mathrm{H} 41$ & $\mathrm{NF}$ & $\mathrm{NF}$ & $\operatorname{mar} A$ & $\mathrm{NF}$ & $\mathrm{NF}$ & $\mathrm{NF}$ & $q n r B 19$ & $\mathrm{NF}$ & $\mathrm{NF}$ & $\mathrm{NF}$ & 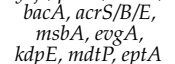 & No & No & $\mathrm{Col}$ \\
\hline BN50 & $\mathrm{O}^{*}: \mathrm{H} 4$ & 167 & $\mathrm{ND}$ & $\mathrm{H} 30$ & NF & $\mathrm{NF}$ & $\operatorname{mar} A$ & $\mathrm{NF}$ & $\mathrm{NF}$ & $\mathrm{NF}$ & $\mathrm{NF}$ & sul2 & $\mathrm{NF}$ & dfra14 & $\begin{array}{l}\text { bapes } \\
\text { batstA }\end{array}$ & No & Yes & $\underset{\text { IncHI1A }}{\mathrm{NF}}$ \\
\hline BN55 & $\mathrm{O} 185: \mathrm{H} 8$ & 155 & $\mathrm{ND}$ & $\mathrm{H} 30$ & $\begin{array}{l}\operatorname{acrB}, \text {, aadA1, } \\
\operatorname{aac}(3)-\mathrm{lb}, \\
\operatorname{aac}\left(6^{\prime}\right) \mathrm{bl} 7\end{array}$ & $\mathrm{NF}$ & $\operatorname{mar} A$ & $b l_{\mathrm{TEM}-220}$ & NF & $\mathrm{NF}$ & $\mathrm{NF}$ & sul1 & $\operatorname{tet}(A)$ & $d f r A 1$ & 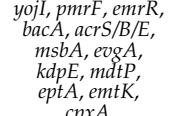 & Yes & Yes & $\begin{array}{l}\text { IncFIB, } \\
\text { IncQ1 }\end{array}$ \\
\hline BN56 & O*:H7 & 2163 & ND & $\mathrm{H} 30$ & $\mathrm{NF}$ & $\mathrm{NF}$ & $\operatorname{mar} A$ & $\mathrm{NF}$ & $\mathrm{NF}$ & NF & $\mathrm{NF}$ & $\mathrm{NF}$ & $\mathrm{NF}$ & $\mathrm{NF}$ & emrR, baes & No & No & $\mathrm{NF}$ \\
\hline $\begin{array}{l}\text { BN57 } \\
\text { BN6 }\end{array}$ & O6:H1 & 73 & $\mathrm{ND}$ & $\begin{array}{l}\mathrm{H} 30 \\
\mathrm{H} 22\end{array}$ & $\operatorname{aac}(3)-\mathrm{Ib}$ & $\mathrm{NF}$ & $\begin{array}{l}\operatorname{marA} A \\
\operatorname{mar} A\end{array}$ & $b^{b l a \mathrm{TEM}-220}$ & $\mathrm{NF}$ & $\mathrm{NF}$ & NF & $\begin{array}{l}\text { Sul2 } \\
\text { sul1 }\end{array}$ & 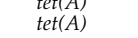 & $\frac{N F}{d f r} A 7$ & $\begin{array}{l}\text { matt, emrK } \\
\text { NF }\end{array}$ & $\begin{array}{l}\text { No } \\
\text { Yes }\end{array}$ & $\begin{array}{l}\text { Yes } \\
\text { Yes }\end{array}$ & IncFIB \\
\hline
\end{tabular}

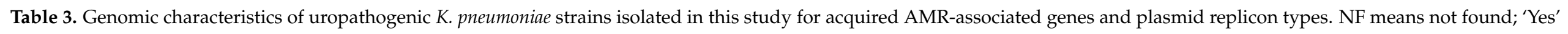
means the strain is either ESBL-producing or MDR; and 'No' means the sample is either non-ESBL or non-MDR.

\begin{tabular}{|c|c|c|c|c|c|c|c|c|c|c|c|c|c|c|c|c|}
\hline $\begin{array}{l}\text { Sample } \\
\text { Name }\end{array}$ & $\begin{array}{c}\text { Sequence } \\
\text { Type } \\
\text { Pasteur }\end{array}$ & $\begin{array}{c}\text { AMR } \\
\text { Phenotype }\end{array}$ & Aminoglycosides & Ciplrofloxacin & $\begin{array}{c}\text { Penicillins }+ \\
\beta \text {-lactamase } \\
\text { Inhibitors }\end{array}$ & $\begin{array}{c}\text { ESBL } \\
\text { Cephalosporins }\end{array}$ & Phenicols & Fluoroquinolones & Quinolone & Sulfonamide & Tetracycline & $\begin{array}{c}\text { Folate } \\
\text { Pathway } \\
\text { Inhibitors }\end{array}$ & $\begin{array}{l}\text { Antibiotic Ef- } \\
\text { fulx/Regulation }\end{array}$ & ESBL & MDR & $\begin{array}{l}\text { Plasmid } \\
\text { Replicon }\end{array}$ \\
\hline 25 & 11 & ND & $\begin{array}{c}\operatorname{aac}\left(6^{\prime}\right)-30 / \operatorname{aac}\left(6^{\prime}\right)- \\
\mathrm{I} \mathrm{b}^{\prime} / 7 / 1 / 10 \\
\operatorname{aad} A 9\end{array}$ & $\mathrm{NF}$ & $b l a_{\mathrm{LEN}-4 / 6}$ & 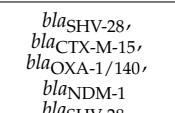 & catB3 & $\begin{array}{c}\text { oqxB, } \\
\operatorname{aac}\left(6^{\prime}\right)-\mathrm{Ib}-\mathrm{cr}\end{array}$ & $q n r B 9$ & sul2 & NF & $\mathrm{NF}$ & $\mathrm{NF}$ & Yes & Yes & $\begin{array}{l}\text { IncFII, } \\
\text { IncFIB, IncR }\end{array}$ \\
\hline 27 & 11 & ND & $\begin{array}{c}\operatorname{aac}\left(6^{\prime}\right)-30 / \operatorname{aac}\left(6^{\prime}\right)- \\
\mathrm{Ib}^{\prime} / 7 / 1 / 10 \\
\operatorname{aad} A 9\end{array}$ & $\mathrm{NF}$ & $b l a_{\mathrm{LEN}-4 / 6}$ & 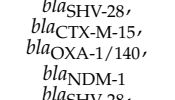 & cat $B 3$ & $\begin{array}{c}\operatorname{oq} \times B, \\
\operatorname{aac}\left(6^{\prime}\right)-\mathrm{Ib}-\mathrm{cr}\end{array}$ & qnrB9 & sul2 & NF & $\mathrm{NF}$ & $\mathrm{NF}$ & Yes & Yes & $\begin{array}{l}\text { IncFII, } \\
\text { IncFIB, IncR }\end{array}$ \\
\hline 29 & 11 & ND & $\begin{array}{c}\operatorname{aac}\left(6^{\prime}\right)-30 / \operatorname{aac}\left(6^{\prime}\right)- \\
\mathrm{Ib}^{\prime} / 7 / 10 \\
\text { aad } A 9\end{array}$ & $\mathrm{NF}$ & $b l a_{\mathrm{LEN}-4 / 6}$ & 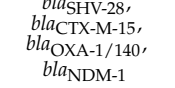 & cat $B 3$ & $\begin{array}{c}\operatorname{oqx} \times, \\
\operatorname{aac}\left(6^{\prime}\right)-\mathrm{Ib}-\mathrm{cr}\end{array}$ & $q n r B 9$ & sul2 & $\mathrm{NF}$ & $\mathrm{NF}$ & $\mathrm{NF}$ & Yes & Yes & $\begin{array}{l}\text { IncFII, } \\
\text { IncFIB, IncR }\end{array}$ \\
\hline
\end{tabular}


Table 3. Cont.

\begin{tabular}{|c|c|c|c|c|c|c|c|c|c|c|c|c|c|c|c|c|}
\hline $\begin{array}{l}\text { Sample } \\
\text { Name }\end{array}$ & $\begin{array}{l}\text { Sequence } \\
\text { Type } \\
\text { Pasteur }\end{array}$ & $\begin{array}{c}\text { AMR } \\
\text { Phenotype }\end{array}$ & Aminoglycosides & Ciplrofloxacin & $\begin{array}{l}\text { Penicillins + } \\
\beta \text {-lactamase } \\
\text { Inhibitors }\end{array}$ & $\begin{array}{c}\text { ESBL } \\
\text { Cephalosporins }\end{array}$ & Phenicols & Fluoroquinolones & Quinolone & Sulfonamide & Tetracycline & $\begin{array}{c}\text { Folate } \\
\text { Pathway } \\
\text { Inhibitors }\end{array}$ & $\begin{array}{l}\text { Antibiotic Ef- } \\
\text { fulx/Regulation }\end{array}$ & ESBL & MDR & $\begin{array}{l}\text { Plasmid } \\
\text { Replicon }\end{array}$ \\
\hline 30 & 11 & ND & $\begin{array}{c}\operatorname{aac}\left(6^{\prime}\right)-30 / \operatorname{aac}\left(6^{\prime}\right)- \\
\mathrm{Ib}^{\prime} / 7 / 10 \\
\operatorname{aad} A 9\end{array}$ & $\mathrm{NF}$ & $b a_{\mathrm{LEN}-4 / 6}$ & $\begin{array}{c}\text { bla }_{\text {SHV-28, }} \\
\text { bla }_{\text {CTX-M-15 }} \\
\text { bla }_{\text {OXA-1/140 }} \\
\text { bla }_{\text {NDM-1 }}\end{array}$ & catB3 & $\begin{array}{c}o q x B, \\
\operatorname{aac}\left(6^{\prime}\right)-\mathrm{Ib}-\mathrm{cr}\end{array}$ & $q n r B 9$ & sul2 & $\mathrm{NF}$ & $\mathrm{NF}$ & $\mathrm{NF}$ & Yes & Yes & $\begin{array}{c}\text { IncFII, } \\
\text { IncFIB, IncR }\end{array}$ \\
\hline 31 & 11 & ND & $\begin{array}{c}\operatorname{aac}\left(6^{\prime}\right)-30 / \operatorname{aac}\left(6^{\prime}\right)- \\
\mathrm{Ib}^{\prime} / 7 / 1 / 10 \\
\text { aad } A 9\end{array}$ & $\mathrm{NF}$ & $b l a_{\mathrm{LEN}-4 / 6}$ & 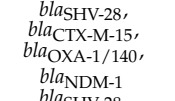 & catB3 & $\begin{array}{c}o q x B, \\
\operatorname{aac}\left(\sigma^{\prime}\right)-\mathrm{rb}-\mathrm{cr}\end{array}$ & qnrB9 & sul2 & $\mathrm{NF}$ & $\mathrm{NF}$ & $\mathrm{NF}$ & Yes & Yes & $\begin{array}{c}\text { IncFII, } \\
\text { IncFIB, IncR }\end{array}$ \\
\hline 32 & 11 & ND & $\begin{array}{c}\operatorname{aac}\left(6^{\prime}\right)-30 / \operatorname{aac}\left(6^{\prime}\right)- \\
\mathrm{Ib}^{\prime} / 7 / 10 \\
\text { aad } A 9\end{array}$ & $\mathrm{NF}$ & $b l a_{\mathrm{LEN}-4 / 6}$ & $\begin{array}{c}\text { bla }_{\text {SHV-28, }} \\
\text { bla }_{\text {CTX-M-15 }} \\
\text { bla }_{\text {OXA-1/140, }} \\
\text { bla }_{\text {NDM-1 }}\end{array}$ & catB3 & $\begin{array}{c}\text { oqxB, } \\
\operatorname{aac}\left(6^{\prime}\right)-\mathrm{Ib}-\mathrm{cr}\end{array}$ & qnrB9 & sul2 & $\mathrm{NF}$ & $\mathrm{NF}$ & $\mathrm{NF}$ & Yes & Yes & $\begin{array}{c}\text { IncFII, } \\
\text { IncFIB, IncR }\end{array}$ \\
\hline 34 & 11 & ND & $\begin{array}{c}\operatorname{aac}\left(6^{\prime}\right)-30 / \operatorname{aac}\left(6^{\prime}\right)- \\
\mathrm{Ib}^{\prime} / 7 / 170 \\
\text { aad } A 9\end{array}$ & $\mathrm{NF}$ & $b l a_{\mathrm{LEN}-4 / 6}$ & $\begin{array}{c}\text { bla }_{\mathrm{SHV}-28} \\
\text { bla }_{\text {CTX-M-15, }} \\
\text { bla }_{\mathrm{OXA}-1 / 140} \\
\text { bla }_{\mathrm{NDM}-1}\end{array}$ & catB3 & $\begin{array}{c}\text { oqxB, } \\
\operatorname{aac}\left(6^{\prime}\right) \text {-Ib-cr }\end{array}$ & qnrB9 & sul2 & $\mathrm{NF}$ & $\mathrm{NF}$ & $\mathrm{NF}$ & Yes & Yes & $\begin{array}{c}\text { IncFII, } \\
\text { IncFIB, IncR }\end{array}$ \\
\hline 35 & 11 & ND & $\begin{array}{c}\operatorname{aac}\left(6^{\prime}\right)-30 / \operatorname{aac}\left(6^{\prime}\right)- \\
\mathrm{Ib}^{\prime} / 7 / 10 \\
\operatorname{aad} A 9\end{array}$ & $\mathrm{NF}$ & $b l a_{\mathrm{LEN}-4 / 6}$ & $\begin{array}{c}\text { bla } \\
\text { bla } \text { CTX-28, }_{\text {CTX-15, }} \\
\text { bla }_{\text {OXA-1/140, }} \\
\text { bla }_{\text {NDM-1 }}\end{array}$ & catB3 & $\begin{array}{c}\text { oqxB, } \\
\text { aac }\left(6^{\prime}\right)-\mathrm{Ib}-\mathrm{cr}\end{array}$ & qnrB9 & sul2 & $\mathrm{NF}$ & $\mathrm{NF}$ & $\mathrm{NF}$ & Yes & Yes & $\begin{array}{l}\text { IncFIII, } \\
\text { IncFIB, IncR }\end{array}$ \\
\hline 36 & 11 & ND & $\begin{array}{c}\operatorname{aac}\left(6^{\prime}\right)-30 / \operatorname{aac}\left(6^{\prime}\right)- \\
\mathrm{Ib} / 7 / 10 \\
\operatorname{aad} A 9\end{array}$ & $\mathrm{NF}$ & $b l a_{\mathrm{LEN}-4 / 6}$ & 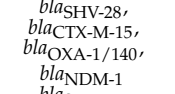 & catB3 & $\begin{array}{c}\text { oqxB, } \\
\operatorname{aac}\left(6^{\prime}\right) \text {-Ib-cr }\end{array}$ & qnrB9 & sul2 & $\mathrm{NF}$ & $\mathrm{NF}$ & $\mathrm{NF}$ & Yes & Yes & $\begin{array}{l}\text { IncFII, } \\
\text { IncFIB, IncR }\end{array}$ \\
\hline 55 & 11 & ND & $\begin{array}{c}\operatorname{aac}\left(6^{\prime}\right)-30 / \operatorname{aac}\left(6^{\prime}\right)- \\
\mathrm{Ib}^{\prime} / 7 / 10 \\
\operatorname{aad} A 9\end{array}$ & $\mathrm{NF}$ & $b a_{\mathrm{LEN}-4 / 6}$ & $\begin{array}{c}\text { bla }_{\text {SHV-28, }} \\
\text { bla }_{\text {CTX-M-15 }} \\
\text { bla }_{\text {OXA-1/140, }} \\
\text { bla }_{\text {NDM-1 }}\end{array}$ & catB3 & $\begin{array}{c}\text { oqxB, } \\
\operatorname{aac}\left(6^{\prime}\right) \text {-lb-cr }\end{array}$ & qnrB9 & sul2 & $\mathrm{NF}$ & $\mathrm{NF}$ & $\mathrm{NF}$ & Yes & Yes & $\begin{array}{c}\text { IncFII, } \\
\text { IncFIB, IncR }\end{array}$ \\
\hline 56 & 11 & ND & $\begin{array}{c}\operatorname{aac}\left(6^{\prime}\right)-30 / \operatorname{aac}\left(6^{\prime}\right)- \\
\mathrm{Ib}^{\prime} / 7 / 10 \\
\operatorname{aad} A 9\end{array}$ & $\mathrm{NF}$ & $b a_{\mathrm{LEN}-4 / 6}$ & 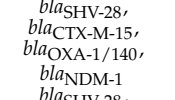 & catB3 & $\begin{array}{c}\text { oqxB, } \\
\text { aac( }\left(6^{\prime}\right) \text {-lb-cr }\end{array}$ & qnrB9 & sul2 & $\mathrm{NF}$ & $\mathrm{NF}$ & $\mathrm{NF}$ & Yes & Yes & $\begin{array}{l}\text { IncFII, } \\
\text { IncFIB, IncR }\end{array}$ \\
\hline 89 & 11 & ND & $\begin{array}{c}\operatorname{aac}\left(6^{\prime}\right)-30 / \operatorname{aac}\left(6^{\prime}\right)- \\
\mathrm{Ib}^{\prime} / 7 / 10 \\
\operatorname{aad} A 9\end{array}$ & $\mathrm{NF}$ & $b l a_{\mathrm{LEN}-4 / 6}$ & 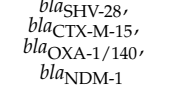 & catB3 & $\begin{array}{c}\text { oqxB, } \\
\operatorname{aac}\left(6^{\prime}\right)-\mathrm{lb}-\mathrm{cr}\end{array}$ & qnrB9 & sul2 & $\mathrm{NF}$ & $\mathrm{NF}$ & $\mathrm{NF}$ & Yes & Yes & $\begin{array}{l}\text { IncFII, } \\
\text { IncFIB, IncR }\end{array}$ \\
\hline 90 & $\mathrm{NF}$ & ND & $\operatorname{aac}\left(6^{\prime}\right)-\mathrm{Ib} 7$, aadA9 & $\mathrm{NF}$ & $b l a_{\mathrm{LEN}-3 / 4 / 5 / 6}$ & $\begin{array}{l}\text { bla } a_{\mathrm{SHV}-28,} \\
b a_{\mathrm{CTX}-\mathrm{M}-15,} \\
{ }^{b l a_{\mathrm{OXX}-1 / 140}}\end{array}$ & $\mathrm{NF}$ & $\mathrm{NF}$ & $\mathrm{NF}$ & sul1 & $\operatorname{tet}(\mathrm{A})$ & dfrA17 & $\begin{array}{l}\text { yojI, pmrF, } \\
\text { emrR, bacA, } \\
\text { acrB, msbA, } \\
\text { evgA, kdpE, } \\
\text { mdtP, eptA, } \\
\text { emtK, cpxA }\end{array}$ & Yes & Yes & IncN \\
\hline BN14 & obse & ND & $\begin{array}{c}\operatorname{aac}\left(6^{\prime}\right)-30 / \operatorname{aac}\left(6^{\prime}\right)- \\
\mathrm{Ib}^{\prime} / 7 / 170 \\
\operatorname{aad} A 9\end{array}$ & marA & $b l a_{\mathrm{LEN}-3 / 4 / 5 / 6}$ & 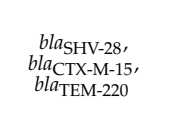 & catB3 & $\begin{array}{c}o g x A / \mathrm{B} \\
\operatorname{aac}\left(6^{\prime}\right)-\mathrm{lb}-\mathrm{cr}\end{array}$ & $q n r B 6 / 17$ & sul1 & $\operatorname{tet}(\mathrm{B})$ & dfrA27 & $\begin{array}{l}\text { yojI, pmrF, } \\
\text { emrR, bacA, } \\
\text { acrB, msbA, } \\
\text { evgA, kdpE, } \\
\text { mdtP, eptA, } \\
\text { emtK, cpxA }\end{array}$ & Yes & Yes & $\begin{array}{l}\text { IncR, IncFII, } \\
\text { IncFIA, Col, } \\
\text { IncX4 }\end{array}$ \\
\hline BN16 & $67 \mathrm{~b} 2$ & ND & $\begin{array}{c}\operatorname{aac}\left(6^{\prime}\right)-30 / \operatorname{aac}\left(6^{\prime}\right)- \\
\mathrm{Ib}^{\prime} / 7 / 170 \\
\operatorname{aad} A 9\end{array}$ & $\operatorname{mar} A$ & $b l a_{\mathrm{LEN}-3 / 4 / 5 / 6}$ & $b l a_{\text {CTX-M-15/88 }}$ & catB3 & $\begin{array}{c}o q x A / \mathrm{B}, \\
\operatorname{aac}\left(6^{\prime}\right)-\mathrm{lb}-\mathrm{cr}\end{array}$ & $q n r B 6 / 17$ & sul1, sul2 & $\operatorname{tet}(\mathrm{B})$ & dfrA27 & $\begin{array}{l}\text { yojI, pmrF, } \\
\text { emrR, bacA, } \\
\text { acrB, msbA, } \\
\text { evgA, kdpE, } \\
\text { mdtP, eptA, } \\
\text { emtK, cpxA }\end{array}$ & Yes & Yes & $\begin{array}{l}\text { IncR, IncFII, } \\
\text { IncFIA, Col, } \\
\text { IncX4 }\end{array}$ \\
\hline BN7 & $6 \mathrm{~b} 6 \mathrm{f}$ & ND & $\begin{array}{c}\operatorname{aac}\left(6^{\prime}\right)-30 / a a c\left(6^{\prime}\right)- \\
\mathrm{Ib}^{\prime} / 7 / 170 \\
\text { aad } A 9\end{array}$ & $\mathrm{NF}$ & $\mathrm{NF}$ & $\mathrm{NF}$ & $\mathrm{NF}$ & $\begin{array}{c}o q x A / \mathrm{B} \\
\operatorname{aac}\left(6^{\prime}\right)-\mathrm{Ib}-\mathrm{cr}\end{array}$ & $q n r B 6 / 17$ & $\mathrm{NF}$ & $\mathrm{NF}$ & $\mathrm{NF}$ & $\mathrm{NF}$ & No & Yes & IncR \\
\hline
\end{tabular}


All $n=19 \mathrm{~K}$. pneumoniae isolates were resistant to aminoglycosides and had either $\operatorname{aac}\left(6^{\prime}\right)-30 / \operatorname{aac}\left(6^{\prime}\right)$-Ib' $/ 7 / 10$-aadA9 $(n=10 / 19)$ or aac $\left(6^{\prime}\right)$-Ib7-aadA9 $(n=1 / 19)$ combination. $\mathrm{N}=18 / 19$ are potentially sulfonamide insusceptible and contained either sul1 only $(n=2 / 19)$, sul2 only $(n=15 / 19)$ or both $(n=1)$. The $\beta$-lactamase $b l a_{\text {LEN }}$ gene is present in all but the BN7 strain, with $b l a_{\mathrm{LEN}-4 / 6}\left(n=15\right.$ from KY) or $b l a_{\mathrm{LEN}-3 / 4 / 5 / 6}$ alleles. BN7 was also the only susceptible isolate against ESBL cephalosphorins. The rest are ESBL producers: $n=15 / 19$ had $b l a_{\mathrm{SHV}-28}, b l a_{\mathrm{CTX}-\mathrm{M}-15}, b l a_{\mathrm{OXA}-1 / 140}$ and $b l a_{\mathrm{NDM}-1}, n=1 / 19$ were observed with the $b l a_{\mathrm{SHV}-28-\mathrm{CTX}-\mathrm{M}-15-\mathrm{OXA}-1 / 140}$ combination and $n=1 / 19$ had $b l a_{\mathrm{SHV}-28}, b l a_{\mathrm{CTX}-\mathrm{M}-15 / 88}$ only. All $n=3$ strains from UG had tet(B) and $d f r A$ (17 or 27 allele type); these strains also contained efflux pump-expressing genes: yojI, $\operatorname{pmrF}, \operatorname{emr} R, \operatorname{bac} A, \operatorname{acr} B, m s b A, \operatorname{evg} A$, $k d p E, m d t P, e p t A, e m t K$, and $c p x A . \mathrm{N}=2 / 19$ (BN14 and BN16 from UG) had ciprofloxacinresistance gene, mar $A$. Only the strain 90 from $\mathrm{KY}$ was not resistant to phenicols, while the rest were detected with the cat gene, specifically, catB3 (Table 3; Supplementary Figure S2b). Overall, $80 \%$ of our E. coli uropathogens had ESBL genes ( $n=15$ strains from UG and $n=29$ from KY) and 93\% of these UPEC are MDR, while all the K. pneumoniae isolates are MDR and only $n=1$ out of the total $n=19(95 \%)$ are ESBL.

\subsection{Population Structure of KY and UG Uropathogens}

The UPEC collection was polyclonal. Eighteen (18) different sequence types were identified in the UPEC population (Achtman scheme). The most prevalent MLST sequence types were ST131 $(n=17 / 55,31 \%)$, ST335 and ST1193 $(n=6 / 55,11 \%)$ and ST10 $(n=4 / 55,7 \%)$. These sequence types were usually associated with UTI cases (Nicolas-Chanoine et al. 2014; Afset et al. 2008; Yamaji et al. 2018); the globally disseminated ESBL-ST131 stood out to be the most dominant ST. Other clones were also observed: $n=3$ ST73, $n=2$ each from ST155, ST410, ST6161 and ST162, and singletons from ST44, ST48, ST165, ST167, ST212, ST448, ST617, ST648 and ST2163; $n=2$ strains from UG (BN2 and BN48) were unclassified (Figure 1a, Table 2). E. coli isolates from UG belong to 15 STs and were thus more diverse compared to those collected from KY, which belong to only 6 STs (Figure 1a, Table 2). This difference in diversity is consistent with the number of serotypes found in UG relative to those from KY: Ugandan strains belong to 20 different $\mathrm{O}: \mathrm{H}$ antigen combinations while the KYn ones were found to have $9 \mathrm{O}: \mathrm{H}$ types.

Fifteen out of nineteen $(n=15 / 19) \mathrm{K}$. pneumoniae isolates from KY belong to ST11 (Pasteur scheme); $n=3 / 19$ UG had no defined sequence types (BN14: 0b8e, BN16; 67b2, BN7: 6b6f) and formed their own clade (Table 3; Figure 1b).

We compared our E. coli samples from the three most prevalent clones, ST131, ST335 and ST10, and our K. pneumoniae strains with previously published genomes listed in BacWGSTdb 2.0. Based on the metadata of the reference genomes, these strains were of different geographical origins (country/state) and were mostly isolated from human hosts and have caused disease (Supplementary Table S2). Computing for the pairwise SNP distances showed that strain CP023853 is the most closely related genome with our KY isolates with distances ranging from 910-1489; CP023853 was also sampled from a UTI patient in Sweden in 2009 (Supplementary Table S2; Supplementary Figure S2a). Our ST335 collection is solely composed of KY isolates, and all appeared to be genetically distant to the selected sequences in the database with a minimum of 4700 SNP differences between the two groups (Supplementary Figure S2a). In contrast, our E. coli ST10 strains were all from UG. The closest reference isolate was LSBS01 (isolated from a fecal sample; Supplementary Table S2), which was 2009 and 2070 SNPs apart from BN20 and BN70, respectively (Supplementary Figure S2a).

Our K. pneumoniae collection, which was dominated by ST11 showed 3500 SNP differences from strain 27 from KY while those that had no defined ST (e.g., BN14 and BN 16 from UG) appeared to be most closely related (minimum SNP distances of 3519 and 3255 ) to the human isolate references LXMM01 and VUBS01, respectively (Supplementary Table S2; Supplementary Figure S2b). 


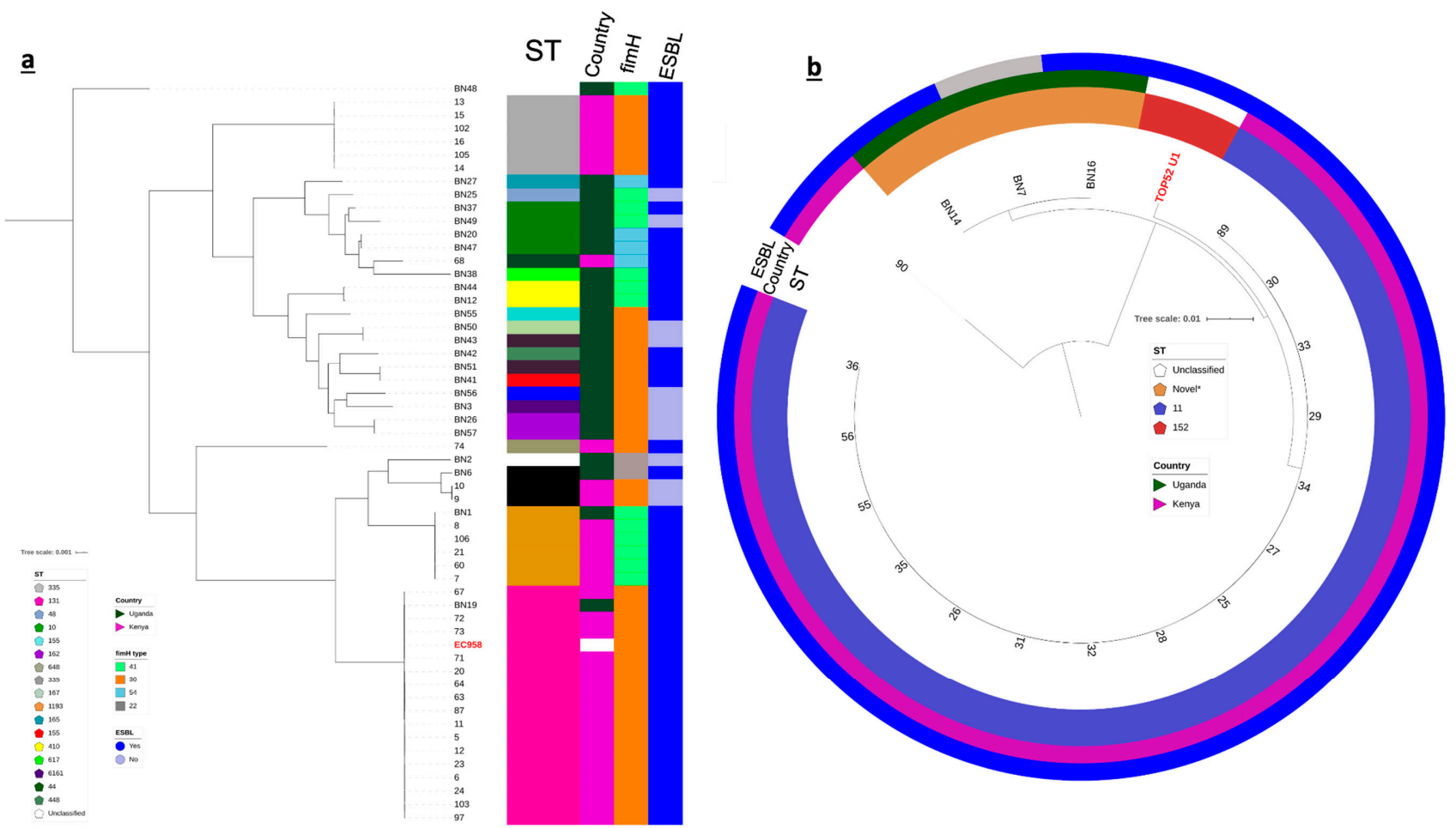

Figure 1. Maximum likelihood phylogenies of core genomes of E. coli (a) and K. pneumoniae (b) uropathogens isolated from KY and UG; reference genomes are in red and font. The mid-point rooted phylograms was constructed using 2862 and 3464 core genes from E. coli and K. pneumoniae populations, respectively and visualized with iTol. The colored strips adjacent to the E. coli phylogeny represent (from left to right) the sequence type (ST), country of origin, type of fimH allele and the ESBL status of each strain. The colored rings around the K. pneumoniae phylogenetic tree indicate the ST, country of origin and the ESBL status of each isolate. "Novel" means the sequence type of the sample/s did not match those in the database and may be novel. The scale bar indicates substitutions per site.

\subsection{Plasmid Characterization}

Genome assemblies of the KY and UG uropathogens were screened for the presence and type of plasmids using PlasmidFinder v.2.1.1. $\mathrm{N}=47 / 55$ in the E. coli collection were found with at least one plasmid. IncFIA was consistently found in $n=10$ had both IncFIA and IncFII, $n=9$ contained IncFIA, IncFII, Col156 types, $n=1$ was detected with IncFIA, IncFII and IncY only or IncI only and IncFII-IncFIA-IncX4 plasmid combinations.

All the samples from the K. pneumoniae collection were found with at least one plasmid type. IncFII-IncFIB-IncR is the most common combination and is found among $n=15$ isolates, while $n=2 / 19$ was found with IncR, IncFII, IncFIA, Col, and IncX4. Notably, the strain 90 from KY had the bla $a_{\mathrm{CTX}-\mathrm{M}-1}$ gene-carrying plasmid IncN and BN7 from UG had the $b l a_{\mathrm{NDM}}$-associated IncR.

\section{Discussion}

We assessed the prevalence of acquired AMR characteristics among uropathogenic E. coli and K. pneumoniae circulating in East African region using WGS. We recruited out-patients that presented UTI-like symptoms from rural areas in KY and UG, which represents a limitation of our sample collection. The lack of point-of-care diagnostic tool such as the use of dipstick test also contributed to some difficulties in our screening. This is evidenced by a high level of contaminants that comprised of strains that do not contain UTI determinants. Nevertheless, our in silico predictions using whole genome analysis revealed alarming rates of ESBL-producing and MDR strains in both our UPEC and K. pneumoniae collections, which reiterates the great necessity for effective interventions to curb their spread. 
Our results firmly indicate a high diversity among E. coli uropathogens, which was more evident in samples taken from UG rather than KY. Strains that belong to the same clonal group had $<200$ core SNPs from each other. This rich genetic diversity is consistent with those observed in other isolates collected from rural or semi-rural communities of low/middle-income countries [47-49]. The widely disseminated UTI-causing clones ST131, ST335 and ST10 were common among our E. coli strains and dominated our Kenyan collection. This is unsurprising as these STs are reported to be circulating globally [50-52]. What is remarkable is the detection of emerging clones such as ST1193 and ST617 that were unusually associated with UTI $[53,54]$ albeit observed in hospital settings. UPEC strains from UG are even more alarming as they represent higher number of unusual or novel UTI clones (i.e., ST155, ST448, and ST162) with potentially higher virulence levels [55-57] compared to those globally-known STs.

Several Klebsiella species were known to have broad-spectrum resistance to common antibiotics [58]. K. pneumoniae strains particularly those belonging to the hypervirulent ST11 have been extensively reported to cause severe infections $[59,60]$ and have led to dire disease outcomes in intensive care units [61]. This K. pneumoniae clone has an alarming antibiotic resistance profile [62] making it difficult to treat. The dominance of ST11 strains in our samples that were mainly collected from outpatients suggests the strong presence of this clinically important bacterial pathogen in the community and pose an apparent threat to public health.

$B l a_{\text {CTX-M }}$ genes were present in $40 \%$ of our UPEC collection and in all but one K. pneumoniae strain (95\%). Notably, the $b a_{\mathrm{CTX}} \mathrm{M}-15$ gene that confers resistance to last-resort antibiotics was found in high levels in both countries. This gene was detected with other ESBL determinants, bla $a_{\mathrm{TEM}}$ and bla $a_{\mathrm{OXA}-1}$ in E. coli and with bla $a_{\mathrm{NDM}}$ in K. pneumoniae, concordant with those in uropathogens found from the Middle East [63] and Asia [64], among others. Consistent with previous findings in other African regions, tet genes in this study were also detected alongside ESBL genes $b l a_{\mathrm{CTX}-\mathrm{M}-15}, b l a_{\mathrm{OXA}-1}$ and $b l a_{\mathrm{TEM}}$ in $n=30 / 55$ E. coli and with $b l a_{\mathrm{LEN}-3 / 4 / 5 / 6}$ among $n=3 / 19 \mathrm{~K}$. pneumoniae $[65,66]$ which stipulates their co-selection and co-transmission in KY and UG. The presence of these genes in the identified plasmid-associated contigs suggest that the mode of transfer may have been plasmid-mediated.

\section{Conclusions}

We underline in this pilot study the high frequency of AMR determinants associated with resistance to common antibiotic classes among E. coli and Klebsiella pneumoniae in East Africa, with specific focus on MDR and ESBL-producing strains from KY and UG. We further demonstrate that routine genomic surveillance is necessary for high-resolution investigation of bacterial epidemiology especially in less represented regions. Our findings have significant implications on improving interventions that aim to address the strong presence of AMR pathogens that cause UTI (particularly in low/middle-income countries).

Supplementary Materials: The following are available online at https://www.mdpi.com/article/10 .3390/antibiotics10121547/s1. Table S1: Metadata of E. coli and K. pneumoniae strains isolated from urine samples including Antibiotic Sensitivity Test results of $n=16$ isolates; Table S2: Comparison of host niche, disease implication, isolation source, collection year, antimicrobial and virulence gene contents between three most abundant E. coli clones, ST131, ST335 and ST10 (a) and K. pneumoniae ST11 (b) strains in our study and selected isolate genomes listed in BacWGSTdb 2.0. ND means not determined; Figure S1: Distribution of antimicrobial resistance genes (AMRGs; right panel) among E. coli (a) and K. pneumoniae (b) isolates from our HATUA Pilot collection. Left panel shows clustering of the strains in a phylogenetic tree according to the presence (green blocks) or absence (pink blocks) of AMRGs; Figure S2: Pairwise SNP distances in core genome multi-locus sequence type (cgMLST)-based alleles of the three most abundant E. coli clones, ST131, ST335, and ST10 (a) and K. pneumoniae ST11 (b) strains in our study and selected isolate genomes listed in BacWGSTdb. The assemblies of the reference sequences were downloaded from European Nucleotide Archive. 
Author Contributions: Conceptualization, S.N.; methodology, A.G.D., K.P., W.S. and M.T.G.H.; software, and M.T.G.H.; validation, H.O., J.B. and K.P.; formal analysis, A.G.D. and M.T.G.H.; investigation, A.G.D.; resources, S.N.; data curation, A.G.D. and M.T.G.H.; writing-original draft preparation, A.G.D.; writing-review and editing, A.G.D., W.S., B.A., J.K., and M.T.G.H.; visualization, A.G.D.; supervision, M.T.G.H.; project administration, M.T.G.H.; funding acquisition, J.K., W.S., B.A., D.J.S. and M.T.G.H. All authors have read and agreed to the published version of the manuscript.

Funding: The Holistic Approach to Unravel Antibacterial Resistance in East Africa is a three-year Global Context Consortia Award (MR/S004785/1) funded by the National Institute for Health Research, Medical Research Council and the Department of Health and Social Care. The award is also part of the EDCTP2 program supported by the European Union.

Institutional Review Board Statement: The study was conducted according to the guidelines of the Declaration of Helsinki, approved by the Institutional Ethics Committee of School of Biomedical Sciences, Makerere University (SBS-HDREC-514 on 13/10/2017) and the Kenya Medical Research Institute (KEMRI) with the approval of KEMRI Scientific and Ethics Review Unit (KEMRI/SERU/CMR/ P00043/3329 on 04 October 2016).

Data Availability Statement: Raw and generated data for this Pilot study are available in the following links: https:/ / doi.org/10.6084/m9.figshare.17105876 (supplementary files, accessed on 16 November 2021), https: / / doi.org/10.6084/m9.figshare.17091293 (draft genome assemblies, accessed on 16 November 2021) and https:/ / doi.org/10.6084/m9.figshare.11965455 (Tables 2 and 3, accessed on16 November 2021).

Acknowledgments: We thank Alison Sandeman, the HATUA Program Manager and Andy Lynch from the Mathematical Institute at the University of St Andrews for their helpful feedback on the manuscript.

Conflicts of Interest: The authors declare no conflict of interest.

\section{References}

1. Michael, C.A.; Dominey-Howes, D.; Labbate, M. The antimicrobial resistance crisis: Causes, consequences, and management. Front. Public Health 2014, 2, 145. [CrossRef] [PubMed]

2. Rather, I.A.; Kim, B.C.; Bajpai, V.K.; Park, Y.H. Self-medication and antibiotic resistance: Crisis, current challenges, and prevention. Saudi J. Biol. Sci. 2017, 24, 808-812. [CrossRef]

3. Nathan, C.; Cars, O. Antibiotic resistance-problems, progress, and prospects. N. Engl. J. Med. 2014, 371, 1761-1763. [CrossRef] [PubMed]

4. French, G.L. The continuing crisis in antibiotic resistance. Int. J. Antimicrob. Agents 2010, 36 (Suppl. 3), S3-S7. [CrossRef]

5. Read, A.F.; Woods, R.J. Antibiotic resistance management. Evol. Med. Public Health 2014, 2014, 147. [CrossRef] [PubMed]

6. Government of Kenya. National Action Plan for the Prevention and Containment of Antimicrobial Resistance; Government of Kenya: Nairobi, Kenya, 2017.

7. UNAS; CDDEP; GARP-Uganda; Mpairwe, Y.; Wamala, S. Antibiotic Resistance in Uganda: Situation Analysis and Recommendations; Uganda National Academy of Sciences: Kampala, Uganda; Center for Disease Dynamics, Economics \& Policy: Washington, DC, USA, 2015; p. 107.

8. Flores-Mireles, A.L.; Walker, J.N.; Caparon, M.; Hultgren, S.J. Urinary tract infections: Epidemiology, mechanisms of infection and treatment options. Nat. Rev. Microbiol. 2015, 13, 269-284. [CrossRef] [PubMed]

9. Kunin, C.M.; Zacha, E.; Paquin, A.J. Urinary-tract infections in schoolchildren. I. Prevalence of bacteriuria and associated urologica. N. Engl. J. Med. 1962, 266, 1287-1296. [CrossRef]

10. Lindberg, U.; Hanson, L.A.; Jodal, U.; Lidin-Janson, G.; Lincoln, K.O.; lling, S. Asymptomatic bacteriuria in schoolgirls. II. Differences in Escherichia coli causing asymptomatic bacteriuria. Acta Paediatr. Scand. 1975, 64, 432-436. [CrossRef]

11. Najar, M.S.; Saldanha, C.L.; Banday, K.A. Approach to urinary tract infections. Indian J. Nephrol. 2009, 19, 129-139. [CrossRef]

12. Levison, M.E.; Kaye, D. Treatment of complicated urinary tract infections with an emphasis on drug-resistant gram-negative uropathogens. Curr. Infect. Dis. Rep. 2013, 15, 109-115. [CrossRef]

13. Nicolle, L.E. Pivmecillinam in the treatment of urinary tract infections. J. Antimicrob. Chemother. 2000, 46 (Suppl. A), 35-39. [CrossRef] [PubMed]

14. Croucher, N.J.; Didelot, X. The application of genomics to tracing bacterial pathogen transmission. Curr. Opin. Microbiol. 2015, 23, 62-67. [CrossRef]

15. Goswami, C.; Fox, S.; Holden, M.; Connor, M.; Leanord, A.; Evans, T.J. Genetic analysis of invasive Escherichia coli in Scotland reveals determinants of healthcare-associated versus community-acquired infections. Microb. Genom. 2018, 4, e000190. [CrossRef] 
16. Argimón, S.; Masim, M.; Gayeta, J.M.; Lagrada, M.L.; Macaranas, P.; Cohen, V.; Limas, M.T.; Espiritu, H.O.; Palarca, J.C.; Chilam, J.; et al. Integrating whole-genome sequencing within the National Antimicrobial Resistance Surveillance Program in the Philippines. Nat. Commun. 2020, 11, 2719. [CrossRef]

17. Halachev, M.R.; Chan, J.Z.-M.; Constantinidou, C.I.; Cumley, N.; Bradley, C.; Smith-Banks, M.; Oppenheim, B.; Pallen, M.J. Genomic epidemiology of a protracted hospital outbreak caused by multidrug-resistant Acinetobacter baumannii in Birmingham, England. Genome Med. 2014, 6, 61070. [CrossRef] [PubMed]

18. Lee, R.S.; Seemann, T.; Heffernan, H.; Kwong, J.C.; Gonçalves da Silva, A.; Carter, G.P.; Woodhouse, R.; Dyet, K.H.; Bulach, D.M.; Stinear, T.P.; et al. Genomic epidemiology and antimicrobial resistance of Neisseria gonorrhoeae in New Zealand. J. Antimicrob. Chemother. 2018, 73, 353-364. [CrossRef]

19. Ludden, C.; Decano, A.G.; Jamrozy, D.; Pickard, D.; Morris, D.; Parkhill, J.; Peacock, S.J.; Cormican, M.; Downing, T. Genomic surveillance of Escherichia coli ST131 identifies local expansion and serial replacement of subclones. Microb. Genom. 2020, 6, e000352. [CrossRef]

20. Masim, M.L.; Argimón, S.; Espiritu, H.O.; Magbanua, M.A.; Lagrada, M.; Olorosa, A.M.; Cohen, V.; Gayeta, J.M.; Jeffrey, B.; Abudahab, K.; et al. Genomic surveillance of methicillin-resistant Staphylococcus aureus in the Philippines, 2013-2014. West. Pac. Surveill. Response J. 2021, 12, 6-16. [CrossRef]

21. Feldgarden, M.; Brover, V.; Haft, D.H.; Prasad, A.B.; Slotta, D.J.; Tolstoy, I.; Tyson, G.H.; Zhao, S.; Hsu, C.H.; McDermott, P.F.; et al. Validating the AMRFinder tool and resistance gene database by using antimicrobial resistance genotype-phenotype correlations in a collection of isolates. Antimicrob. Agents Chemother. 2019, 63, e00483-19. [CrossRef]

22. Chen, S.; Zhou, Y.; Chen, Y.; Gu, J. Fastp: An ultra-fast all-in-one FASTQ preprocessor. Bioinformatics 2018, 34, i884-i890. [CrossRef]

23. Wick, R.R.; Judd, L.M.; Gorrie, C.L.; Holt, K.E. Unicycler: Resolving bacterial genome assemblies from short and long sequencing reads. PLoS Comput. Biol. 2017, 13, e1005595. [CrossRef]

24. Ponstingl, H.; Ning, Z. SMALT-A New Mapper for DNA Sequencing Reads. F1000 Research 2010. Available online: https: / / f1000research.com/posters/327 (accessed on 18 December 2019).

25. Li, H.; Handsaker, B.; Wysoker, A.; Fennell, T.; Ruan, J.; Homer, N.; Marth, G.; Abecasis, G.; Durbin, R.; 1000 Genome Project Data Processing Subgroup. The sequence alignment/map format and SAMtools. Bioinformatics 2009, 25, 2078-2079. [CrossRef]

26. Wylie, K.M.; Wylie, T.N.; Minx, P.J.; Rosen, D.A. Whole-genome sequencing of Klebsiella pneumoniae isolates to track strain progression in a single patient with recurrent urinary tract infection. Front. Cell. Infect. Microbiol. 2019, 9, 14. [CrossRef]

27. Forde, B.M.; Ben Zakour, N.L.; Stanton-Cook, M.; Phan, M.-D.; Totsika, M.; Peters, K.M.; Chan, K.G.; Schembri, M.A.; Upton, M.; Beatson, S.A. The complete genome sequence of Escherichia coli EC958: A high quality reference sequence for the globally disseminated multidrug resistant E. coli O25b:H4-ST131 clone. PLoS ONE 2014, 9, e104400. [CrossRef]

28. Centre for Genomic Pathogen Surveillance (CGPS). Pathogenwatch Private Metadata. Available online: https://cgps.gitbook.io/ pathogenwatch/how-to-use-pathogenwatch/private-metadata (accessed on 31 October 2019).

29. Ondov, B.D.; Treangen, T.J.; Melsted, P.; Mallonee, A.B.; Bergman, N.H.; Koren, S.; Phillippy, A.M. Mash: Fast genome and metagenome distance estimation using MinHash. Genome Biol. 2016, 17, 132. [CrossRef]

30. Tatusova, T.; DiCuccio, M.; Badretdin, A.; Chetvernin, V.; Nawrocki, E.P.; Zaslavsky, L.; Lomsadze, A.; Pruitt, K.D.; Borodovsky, M.; Ostell, J. NCBI prokaryotic genome annotation pipeline. Nucleic Acids Res. 2016, 44, 6614-6624. [CrossRef] [PubMed]

31. Inouye, M.; Dashnow, H.; Raven, L.A.; Schultz, M.B.; Pope, B.J.; Tomita, T.; Zobel, J.; Holt, K.E. SRST2: Rapid genomic surveillance for public health and hospital microbiology labs. Genome Med. 2014, 6, 90. [CrossRef]

32. Achtman, M.; Wain, J.; Weill, F.X.; Nair, S.; Zhou, Z.; Sangal, V.; Krauland, M.G.; Hale, J.L.; Harbottle, H.; Uesbeck, A.; et al. Multilocus sequence typing as a replacement for serotyping in Salmonella enterica. PLoS Pathog. 2012, 8, e1002776. [CrossRef] [PubMed]

33. Diancourt, L.; Passet, V.; Nemec, A.; Dijkshoorn, L.; Brisse, S. The population structure of Acinetobacter baumannii: Expanding multiresistant clones from an ancestral susceptible genetic pool. PLoS ONE 2010, 5, e10034. [CrossRef]

34. Scheutz, F.; Teel, L.D.; Beutin, L.; Pierard, D.; Buvens, G.; Karch, H.; Mellmann, A.; Caprioli, A.; Tozzoli, R.; Morabito, S.; et al. Multicenter evaluation of a sequence-based protocol for subtyping Shiga toxins and standardizing Stx nomenclature. J. Clin. Microbiol. 2012, 50, 2951-2963. [CrossRef]

35. Seemann, T. Prokka: Rapid prokaryotic genome annotation. Bioinformatics 2014, 30, 2068-2069. [CrossRef]

36. McArthur, A.G.; Waglechner, N.; Nizam, F.; Yan, A.; Azad, M.A.; Baylay, A.J.; Bhullar, K.; Canova, M.J.; De Pacale, G.; Ejim, L.; et al. The comprehensive antibiotic resistance database. Antimicrob. Agents Chemother. 2013, 57, 3348-3357. [CrossRef] [PubMed]

37. Feng, Y.; Zou, S.; Chen, H.; Yu, Y.; Ruan, Z. BacWGSTdb 2.0: A one-stop repository for bacterial whole-genome sequence typing and source tracking. Nucleic Acids Res. 2021, 49, D644-D650. [CrossRef]

38. Hadfield, J.; Croucher, N.J.; Goater, R.J.; Abudahab, K.; Aanensen, D.M.; Harris, S.R. Phandango: An interactive viewer for bacterial population genomics. Bioinformatics 2018, 34, 292-293. [CrossRef]

39. Carattoli, A.; Zankari, E.; García-Fernández, A.; Larsen, M.V.; Lund, O.; Villa, L.; Aarestrup, F.M.; Hasman, H. In silico detection and typing of plasmids using PlasmidFinder and plasmid multilocus sequence typing. Antimicrob Agents Chemother. 2014, 58, 3895-3903. [CrossRef]

40. Clinical and Laboratory Standards Institute. Reference Method for Broth Dilution Antifungal Susceptibility Testing of Filamentous Fungi, 30th ed.; Approved Standard; CLSI Document C24-Ed4; Clinical and Laboratory Standards Institute: Wayne, PA, USA, 2016. 
41. Didelot, X.; Wilson, D.J. ClonalFrameML: Efficient inference of recombination in whole bacterial genomes. PLoS Comput. Biol. 2015, 11, e1004041. [CrossRef] [PubMed]

42. Stamatakis, A. RAxML version 8: A tool for phylogenetic analysis and post-analysis of large phylogenies. Bioinformatics 2014, 30, 1312-1313. [CrossRef]

43. Letunic, I.; Bork, P. Interactive Tree of Life (iTOL) v4: Recent updates and new developments. Nucleic Acids Res. 2019, 47, W256-W259. [CrossRef]

44. Rambaut, A. FigTree v1.3.1; Institute of Evolutionary Biology, University of Edinburgh: Edinburgh, Scotland, 2010; Available online: http:/ / tree.bio.ed.ac.uk/software/figtree/ (accessed on 20 January 2020).

45. Page, A.J.; Cummins, C.A.; Hunt, M.; Wong, V.K.; Reuter, S.; Holden, M.T.; Fookes, M.; Falush, D.; Keane, J.A.; Parkhill, J. Roary: Rapid large-scale prokaryote pan genome analysis. Bioinformatics 2015, 31, 3691-3693. [CrossRef]

46. Katoh, K.; Standley, D.M. MAFFT multiple sequence alignment software version 7: Improvements in performance and usability. Mol. Biol. Evol. 2013, 30, 772-780. [CrossRef]

47. Salinas, L.; Cárdenas, P.; Johnson, T.J.; Vasco, K.; Graham, J.; Trueba, G. Diverse commensal Escherichia coli clones and plasmids disseminate antimicrobial resistance genes in domestic animals and children in a semirural community in Ecuador. mSphere 2019, 4, e00316-19. [CrossRef] [PubMed]

48. Kimata, K.; Shima, T.; Shimizu, M.; Tanaka, D.; Isobe, J.; Gyobu, Y.; Watahiki, M.; Nagai, Y. Rapid categorization of pathogenic Escherichia coli by multiplex PCR. Microbiol. Immunol. 2005, 49, 485-492. [CrossRef] [PubMed]

49. Montealegre, M.C.; Roy, S.; Boni, F.; Hossain, M.I.; Navab-Daneshmand, T.; Caduff, L.; Faruque, A.S.G.; Islam, M.A.; Julian, T.R. Risk factors for detection, survival, and growth of antibiotic-resistant and pathogenic Escherichia coli in household soils in rural Bangladesh. Appl. Environ. Microbiol. 2018, 84, e01978-18. [CrossRef]

50. Ben Zakour, N.L.; Alsheikh-Hussain, A.S.; Ashcroft, M.M.; Khanh, N.T.; Roberts, L.W.; Stanton-Cook, M.; Schembri, M.A.; Beatson, S.A. Sequential acquisition of virulence and fluoroquinolone resistance has shaped the evolution of Escherichia coli ST131. mBio 2016, 7, e00347-16. [CrossRef]

51. Sanjar, F.; Rusconi, B.; Hazen, T.H.; Koenig, S.S.; Mammel, M.K.; Feng, P.C.; Rasko, D.A.; Eppinger, M. Characterization of the pathogenome and phylogenomic classification of enteropathogenic Escherichia coli of the O157: Non-H7 serotypes. Pathog Dis. 2015, 73, ftv033. [CrossRef]

52. Day, M.J.; Hopkins, K.L.; Wareham, D.W.; Toleman, M.A.; Elviss, N.; Randall, L.; Teale, C.; Clearly, P.; Wiuff, C.; Doumith, M.; et al. Extended-spectrum $\beta$-lactamase-producing Escherichia coli in human-derived and foodchain-derived samples from England, Wales, and Scotland: An epidemiological surveillance and typing study. Lancet. Infect. Dis. 2019, 19, 1325-1335. [CrossRef]

53. Valenza, G.; Werner, M.; Eisenberger, D.; Nickel, S.; Lehner-Reindl, V.; Holler, C.; Bogdan, C. First report of the new emerging global clone ST1193 among clinical isolates of extended-spectrum $\beta$-lactamase (ESBL)-producing Escherichia coli from Germany. J. Glob. Antimicrob. Resist. 2019, 17, 305-308. [CrossRef] [PubMed]

54. Mhaya, A.; Trabelsi, R.; Begu, D.; Aillerie, S.; M'zali, F.; Tounsi, S.; Gdoura, R.; Arpin, C. Emergence of B2-ST131-C2 and A-ST617 Escherichia coli clones producing both CTX-M-15- and CTX-M-27 and ST147 NDM-1 positive Klebsiella pneumoniae in the Tunisian community. bioRxiv 2019. [CrossRef]

55. Castellanos, L.R.; Donado-Godoy, P.; León, M.; Clavijo, V.; Arevalo, A.; Bernal, J.F.; Timmerman, A.J.; Mevius, D.J.; Wagenaar, J.A.; Hordijk, J. High heterogeneity of Escherichia coli sequence types harbouring ESBL/AmpC genes on IncI1 plasmids in the colombian poultry chain. PLoS ONE 2017, 12, e0170777. [CrossRef]

56. Choudhury, N.A.; Paul, D.; Chakravarty, A.; Bhattacharjee, A.; Dhar Chanda, D. IncX3 plasmid mediated occurrence of blaNDM-4 within Escherichia coli ST448 from India. J. Infect. Public Health 2018, 11, 111-114. [CrossRef]

57. Zhang, P.; Wang, J.; Wang, X.; Bai, X.; Ma, J.; Dang, R.; Xiong, Y.; Fanning, S.; Bai, L.; Yang, Z. Characterization of five Escherichia coli isolates co-expressing ESBL and MCR-1 resistance mechanisms from different origins in China. Front. Microbiol. 2019, 10, 1994. [CrossRef]

58. Ghenea, A.E.; Cioboată, R.; Drocaş, A.I.; Țieranu, E.N.; Vasile, C.M.; Moroşanu, A.; Țieranu, C.G.; Salan, A.I.; Popescu, M.; Turculeanu, A.; et al. Prevalence and antimicrobial resistance of Klebsiella strains isolated from a county hospital in Romania. Antibiotics 2021, 10, 868. [CrossRef]

59. Yang, J.; Ye, L.; Guo, L.; Zhao, Q.; Chen, R.; Luo, Y.; Chen, Y.; Tian, S.; Zhao, J.; Shen, D.; et al. A nosocomial outbreak of KPC-2-producing Klebsiella pneumoniae in a Chinese hospital: Dissemination of ST11 and emergence of ST37, ST392 and ST395. Clin. Microbiol. Infect. 2013, 19, E509-E515. [CrossRef] [PubMed]

60. Li, J.; Zou, M.X.; Wang, H.C.; Dou, Q.Y.; Hu, Y.M.; Yan, Q.; Liu, W.E. An outbreak of infections caused by a Klebsiella pneumoniae ST11 clone coproducing Klebsiella pneumoniae carbapenemase-2 and RmtB in a Chinese Teaching Hospital. Chin. Med. J. 2016, 129, 2033-2039. [CrossRef] [PubMed]

61. Gu, D.; Dong, N.; Zheng, Z.; Lin, D.; Huang, M.; Wang, L.; Chan, E.W.; Shu, L.; Yu, J.; Zhang, R.; et al. A fatal outbreak of ST11 carbapenem-resistant hypervirulent Klebsiella pneumoniae in a Chinese hospital: A molecular epidemiological study. Lancet Infect. Dis. 2018, 18, 37-46. [CrossRef]

62. Jayol, A.; Poirel, L.; Dortet, L.; Nordmann, P. National survey of colistin resistance among carbapenemase-producing Enterobacteriaceae and outbreak caused by colistin-resistant OXA-48-producing Klebsiella pneumoniae, France, 2014. Euro surveillance. Eur. Commun. Dis. Bull. 2016, 21, 30339. [CrossRef] 
63. Alqasim, A.; Abu Jaffal, A.; Alyousef, A.A. Prevalence of multidrug resistance and extended-spectrum $\beta$-lactamase carriage of clinical uropathogenic Escherichia coli isolates in Riyadh, Saudi Arabia. Int. J. Microbiol. 2018, 2018, 3026851. [CrossRef]

64. Zhao, R.; Shi, J.; Shen, Y.; Li, Y.; Han, Q.; Zhang, X.; Gu, G.; Xu, J. Phylogenetic distribution of virulence genes among ESBLproducing uropathogenic Escherichia coli isolated from long-term hospitalized patients. J. Clin. Diagn. Res. 2015, 9, DC01-DC04. [CrossRef] [PubMed]

65. Mbelle, N.M.; Osei Sekyere, J.; Amoako, D.G.; Maningi, N.E.; Modipane, L.; Essack, S.Y.; Feldman, C. Genomic analysis of a multidrug-resistant clinical Providencia rettgeri (PR002) strain with the novel integron $\ln 1483$ and an A/C plasmid replicon. Ann. N. Y. Acad. Sci. 2020, 1462, 92-103. [CrossRef] [PubMed]

66. Adefisoye, M.A.; Okoh, A.I. Identification and antimicrobial resistance prevalence of pathogenic Escherichia coli strains from treated wastewater effluents in Eastern Cape, South Africa. Microbiol. Open 2016, 5, 143-151. [CrossRef] [PubMed] 